\title{
Mean-field density functional theory of a nanoconfined classical, three-dimensional Heisenberg fluid. II. The interplay between molecular packing and orientational order
}

Cite as: J. Chem. Phys. 149, 054704 (2018); https://doi.org/10.1063/1.5040934

Submitted: 22 May 2018 . Accepted: 17 July 2018 . Published Online: 03 August 2018

Stefanie M. Wandrei (D), Roland Roth (D), and Martin Schoen (D)

\section{ARTICLES YOU MAY BE INTERESTED IN}

Mean-field density functional theory of a nanoconfined classical, three-dimensional Heisenberg fluid. I. The role of molecular anchoring

The Journal of Chemical Physics 144, 194704 (2016); https://doi.org/10.1063/1.4949330

Dielectric constant of ionic solutions: Combined effects of correlations and excluded volume

The Journal of Chemical Physics 149, 054504 (2018); https://doi.org/10.1063/1.5042235

A simple closure procedure for the study of velocity autocorrelation functions in fluids as a "bridge" between different theoretical approaches

The Journal of Chemical Physics 149, 054101 (2018); https://doi.org/10.1063/1.5042772

Lock-in Amplifiers Find out more today

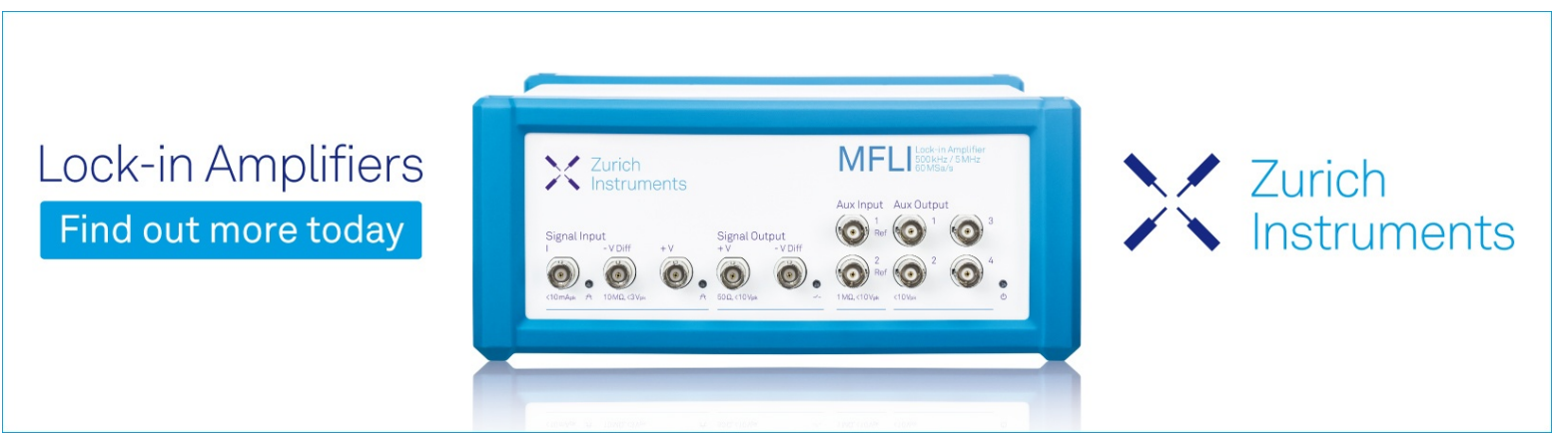

J. Chem. Phys. 149, 054704 (2018); https://doi.org/10.1063/1.5040934 149, 054704 (c) 2018 Author(s). 


\title{
Mean-field density functional theory of a nanoconfined classical, three-dimensional Heisenberg fluid. II. The interplay between molecular packing and orientational order
}

\author{
Stefanie M. Wandrei, ${ }^{1}$ Roland Roth, ${ }^{2}$ and Martin Schoen ${ }^{1,3,4}$ \\ ${ }^{1}$ Stranski-Laboratorium für Physikalische und Theoretische Chemie, Fakultät für Mathematik und \\ Naturwissenschaften, Technische Universität Berlin, Straße des 17. Juni 115, 10623 Berlin, Germany \\ ${ }^{2}$ Institut für Theoretische Physik, Mathematisch-Naturwissenschaftliche Fakultät, Eberhard-Karls-Universität, \\ Auf der Morgenstelle 14, 72076 Tübingen, Germany \\ ${ }^{3}$ Department of Chemical and Biomolecular Engineering, North Carolina State University, Box 7905, \\ 911 Partners Way, Raleigh, North Carolina 27695, USA \\ ${ }^{4}$ Department of Chemical Engineering, Imperial College, South Kensington Campus, London SW7 2AZ, \\ United Kingdom
}

(Received 22 May 2018; accepted 17 July 2018; published online 3 August 2018)

\begin{abstract}
As in Paper I of this series of papers [S. M. Cattes et al., J. Chem. Phys. 144, 194704 (2016)], we study a Heisenberg fluid confined to a nanoscopic slit pore with smooth walls. The pore walls can either energetically discriminate specific orientations of the molecules next to them or are indifferent to molecular orientations. Unlike in Paper I, we employ a version of classical density functional theory that allows us to explicitly account for the stratification of the fluid (i.e., the formation of molecular layers) as a consequence of the symmetry-breaking presence of the pore walls. We treat this stratification within the White Bear version (Mark I) of fundamental measure theory. Thus, in this work, we focus on the interplay between local packing of the molecules and orientational features. In particular, we demonstrate why a critical end point can only exist if the pore walls are not energetically discriminating specific molecular orientations. We analyze in detail the positional and orientational order of the confined fluid and show that reorienting molecules across the pore space can be a twodimensional process. Last but not least, we propose an algorithm based upon a series expansion of Bessel functions of the first kind with which we can solve certain types of integrals in a very efficient manner. Published by AIP Publishing. https://doi.org/10.1063/1.5040934
\end{abstract}

\section{INTRODUCTION}

If a molecule possesses an internal vectorial degree of freedom, the phase behavior of fluidic systems composed of these molecules can be quite complex. A prominent example is the Stockmayer model in which a magnetic point dipole is superimposed on short-range Lennard-Jones interactions between the spherically symmetric cores of the molecules. For bulk Stockmayer fluids, the phase behavior is quite well understood. ${ }^{1-4}$ Unfortunately, the long-range character of the dipole-dipole interaction potential poses a problem in computing its contribution to the overall free energy of the system. ${ }^{1}$

This complication can be avoided if a different model of polar molecules is considered. In this so-called Heisenberg model, the dipole-dipole interaction of the Stockmayer potential is replaced by the interaction between a pair of threedimensional, classical "spins." In the Heisenberg model, all interactions are usually of short-range. As for the Stockmayer model, bulk properties of the Heisenberg model have been extensively studied in the past. ${ }^{5-10}$

In both models, the vectorial degrees of freedom of the molecules allow for the formation of an ordered polar phase besides the more conventional gaseous and isotropic liquid phases. Topologically, three different types of generic phase diagrams exist independent of the short- or long-range character of the orientation dependent part of the interaction potential (see, for example, Ref. 1 for the Stockmayer and Ref. 10 for the Heisenberg model).

For relatively weak coupling between the vectorial degrees of freedom, one observes coexistence between a gas and a polar phase at sufficiently low temperatures. Polar order is lost at a critical end point (cep) from which a line of critical points emerges. Along this critical line, an isotropic liquid phase is transformed into a polar ordered phase during a continuous phase transition. Above the temperature of the cep, one observes coexistence between a gas and an isotropic liquid ending at a critical point at which both phases become indistinguishable.

For intermediate coupling between the vectorial degrees of freedom, one has coexistence between isotropic liquid and polar phases and a triple point at which these latter phases coexist with a gas. The cep then becomes a tricritical point at which again a critical line starts.

For strong coupling, it turns out that the gas-isotropic liquid critical point is suppressed. In this case, a more or less dense fluid coexists with a polar ordered phase all the way up to a higher temperature where again a critical line begins.

These generic types of topologically distinct phase diagrams seem to prevail if instead of the bulk a fluid confined to 
a nanoscopic container is considered. For the Stockmayer fluid, this was first demonstrated by Gramzow and Klapp ${ }^{11}$ and later by Szalai and Dietrich. ${ }^{12}$ In both previous studies ${ }^{11,12}$ (versions of) classical density functional theory (DFT) have been employed. Classical DFT was first presented and discussed in a seminal paper by Evans in $1979 .{ }^{13}$

A crucial assumption made in the work of Gramzow and Klapp ${ }^{11}$ is that the confined fluid is homogeneous as far as the density is concerned despite the layering of fluid molecules that is omnipresent in confinement; only the orientation distribution function is treated as a local quantity. We followed this approach in our earlier publication (henceforth referred to as Paper I). ${ }^{14}$

However, as was demonstrated by Szalai and Dietrich, ${ }^{12}$ a more realistic theory of confined fluids should take into account the inhomogeneity of the fluid in both packing and orientation by also making the density a local quantity. They employ a version of the so-called fundamental measure theory (FMT) originally developed by Rosenfeld. ${ }^{15,16}$

In principle, in FMT, one bases the analysis of the packing on weighted densities where the weight functions are related to geometrical measures of a pair of interacting hard spheres. Weighted density approaches of various kinds exist within the context of DFT. For example, Tarazona and Evans ${ }^{17}$ used the Mayer $f$-function for a pair of hard spheres as a weight function, which Tarazona ${ }^{18}$ refined by adding density dependent weight functions in order to more accurately reproduce the direct correlation function. Similarly Denton and Ashcroft ${ }^{19}$ as well as Leidl and Wagner ${ }^{20}$ used weight functions of various density dependences to describe the hard sphere packing. By contrast, Rosenfeld's version, ${ }^{15,16}$ which was inspired by ideas from scaled particle theory, gives a good agreement with the direct correlation function without the need for density dependent weight functions. Kierlik and Rosinberg $^{21}$ derived a different FMT in which the deconvolution of the Mayer $f$-function employed by them gives a set of density independent weight functions that differs from those of Rosenfeld. ${ }^{15,16}$ However, both approaches are, in fact, equivalent, as was demonstrated a few years later by Phan et al. ${ }^{22}$

As in Paper $\mathrm{I}^{14}$ we confine our Heisenberg fluid to a nanoscopic slit pore with smooth walls. In this study, we follow Szalai and Dietrich ${ }^{12}$ in treating the contribution of the hard-core repulsion to the overall free energy within the framework of FMT. ${ }^{15,16}$ Thus, unlike in Paper $\mathrm{I}^{14}$ of this small series of two papers, we concentrate here on the coupling between the positional structure and orientation effects in a confined Heisenberg fluid. More specifically, we employ the White Bear version (Mark I) of FMT developed by Roth et al. ${ }^{23}$ (see also Ref. 24). The White Bear version of FMT differs from the original FMT proposed by Rosenfeld in which it explicitly uses an equation of state for a multicomponent hard-sphere mixture in the bulk proposed by Mansoori et al. ${ }^{25}$

However, there is a price to pay for the greater realism in describing the orientational and positional structure of confined fluids by FMT. Not unexpectedly, the FMT treatment is computationally much more expensive than assuming a constant density across the pore; this turns out to be a rather limiting factor if full phase diagrams are to be computed more or less routinely. In addition, there are a number of positiondependent integrations over orientations that in a brute force implementation have to be carried out numerically. However, at the core of the numerical procedure, one is dealing with a number of integrals that resemble Bessel functions of the first kind. ${ }^{26}$ One can then employ the series representation of these functions which turns out to converge rapidly resulting in an enormous reduction of computer time. This, in turn, enables us to investigate the coupling between orientational and positional ordering and the impact of this coupling on the phase behavior of a confined Heisenberg fluid.

The remainder of our paper is organized as follows. In Sec. II, we introduce our model system. In Sec. III, we summarize key concepts of our version of classical DFT. Aspects of thermodynamic stability are discussed in Sec. IV. In this section, we also develop a quantitative argument for why only slit pores with walls that do not orient molecules in specific ways can have a phase diagram with a cep; a cep cannot exist for formal reasons if the pore walls are explicitly orienting molecules in specific directions. Section V is devoted to a validation of our model and a presentation of key findings of this study. Our results are finally summarized and discussed in the concluding Sec. VI. In Appendix A, we discuss the squarewell potential in cylindrical coordinates on which this study is based; Appendix B is devoted to the approach based on Bessel functions mentioned above.

\section{MODEL SYSTEM}

\section{A. Fluid phase}

Similar to Paper $\mathrm{I}^{14}$ we focus on a confined fluid consisting of $N$ particles interacting in a pairwise additive fashion via the orientation-dependent potential function $\varphi_{\mathrm{ff}}$. This interaction potential can be decomposed into a potential $\varphi_{\text {iso }}$ describing the interactions between the (spherically symmetric) cores of the particles and a part $\varphi_{\text {anis }}$ referring to the (orientation-dependent) anisotropic interactions. Thus,

$$
\varphi_{\mathrm{ff}}\left(\boldsymbol{r}_{12}, \omega_{1}, \omega_{2}\right)=\varphi_{\text {iso }}\left(r_{12}\right)+\varphi_{\text {anis }}\left(\boldsymbol{r}_{12}, \omega_{1}, \omega_{2}\right),
$$

where $\boldsymbol{r}_{12}=\boldsymbol{r}_{1}-\boldsymbol{r}_{2}$ is the distance vector connecting the centers of the spherically symmetric cores of a pair of particles; these cores are located at $\boldsymbol{r}_{1}$ and $\boldsymbol{r}_{2}$, respectively; $\boldsymbol{r}_{12}=\left|\boldsymbol{r}_{12}\right|$ and $\omega_{i}=\left(\theta_{i}, \phi_{i}\right)(i=1,2)$ are sets of Euler angles describing the orientation of our uniaxially symmetric particles (i.e., their "spins") in a space-fixed frame of reference; $\theta_{i}$ and $\phi_{i}$ are polar and azimuthal angle, respectively.

Unlike in Paper I, ${ }^{14}$ we express $\varphi_{\text {iso }}$ as

$$
\varphi_{\mathrm{iso}}\left(r_{12}\right)=\varphi_{\mathrm{hs}}\left(r_{12}\right)+\varphi_{\mathrm{sw}}\left(r_{12}\right) \text {, }
$$

where $\varphi_{\mathrm{hs}}$ describes the interaction between two hard-spheres of diameter $\sigma$ and

$$
\varphi_{\mathrm{sw}}\left(r_{12}\right)=-\varepsilon \Theta\left(\lambda \sigma-r_{12}\right)
$$

is a superimposed attractive well where the coupling constant $\varepsilon$ determines the attraction strength between the spherically symmetric pair of particles and $\lambda \sigma$ is the width of the attractive trough; throughout this work we take $\lambda=\frac{3}{2}$. Hence, we focus exclusively on short-range attractions. Notice also that 
Eq. (2.3) is consistent with the random phase approximation (RPA) in which the attractive intermolecular interactions are kept constant inside the hard core of the particles. ${ }^{27}$ Within the context of classical DFT, the RPA had been discussed in detail by Archer and Evans ${ }^{28}$ a few years back.

Anisotropic interactions are also taken to be of shortrange. In particular, we adopt

$$
\varphi_{\text {anis }}\left(\boldsymbol{r}_{12}, \omega_{1}, \omega_{2}\right)=-\frac{(4 \pi)^{3 / 2}}{\sqrt{3}} \varepsilon_{\mathrm{H}} \varphi_{\mathrm{sw}}\left(r_{12}\right) \Phi_{110}\left(\omega_{1}, \omega_{2}, \omega\right),
$$

where $\varepsilon_{\mathrm{H}}$ is a (dimensionless) coupling parameter that allows us (at least in principle) to vary the strength of the anisotropic attractions relative to the isotropic ones; $\omega$ is a set of Euler angles specifying the orientation of $\widehat{\boldsymbol{r}}_{12}=\boldsymbol{r}_{12} / r_{12}$ in a spacefixed frame of reference. Notice that here and below the caret indicates a unit vector.

In Eq. (2.4),

$$
\begin{aligned}
\Phi_{l_{1} l_{2} l}\left(\omega_{1}, \omega_{2}, \omega\right)= & \sum_{m_{1}, m_{2}, m} C\left(l_{1}, l_{2}, l ; m_{1}, m_{2}, m\right) \\
& \times \mathcal{Y}_{l_{1} m_{1}}\left(\omega_{1}\right) \mathcal{Y}_{l_{2} m_{2}}\left(\omega_{2}\right) \mathcal{Y}_{l m}^{*}(\omega)
\end{aligned}
$$

is a rotational invariant ${ }^{29}$ where $C$ is a Clebsch-Gordan coefficient, $\mathcal{Y}_{l^{\prime} m^{\prime}}$ is a spherical harmonic, and ${ }^{*}$ indicates the complex conjugate; integers $l^{\prime} \geq 0$ (that is, $l_{1}, l_{2}$, or $l$ ) are related to integers $m^{\prime}$ through the relation $-l^{\prime} \leq m^{\prime} \leq l^{\prime}$ such that for each $l^{\prime}$ there are $2 l^{\prime}+1$ integers $m^{\prime}$ that need to be considered.

For our present model, it is particularly simple to show ${ }^{14}$ that

$$
\begin{aligned}
\Phi_{110}\left(\omega_{1}, \omega_{2}, \omega\right) & =-\frac{\sqrt{3}}{(4 \pi)^{3 / 2}} \cos \gamma \\
& =-\frac{\sqrt{3}}{(4 \pi)^{3 / 2}} \widehat{\boldsymbol{u}}\left(\omega_{1}\right) \cdot \widehat{\boldsymbol{u}}\left(\omega_{1}\right),
\end{aligned}
$$

where $\gamma$ is the angle between the spins of the molecular pair.

\section{B. Confinement to slit-pores with structureless walls}

The model fluid introduced in Sec. II A is confined to a slitpore with plane-parallel, structureless walls that we perceive as an orientation-dependent external field. Specifically,

$$
\widetilde{\Phi}_{\text {ext }}(z, \omega)=\Phi_{\text {ext }}(z, \omega)+\sum_{k=1}^{2} \varphi_{\mathrm{hs}}\left(\Delta z^{(k)}\right),
$$

where $\Delta z^{(k)}=z \pm\left(s_{\mathrm{Z}}-\sigma\right) / 2$ is the distance between the center of mass of a molecule and the plane of a wall. We assume the lower wall $(k=1,+)$ to be located at $-s_{\mathrm{Z}} / 2$, whereas the upper one $(k=2,-)$ is located at $+s_{\mathrm{Z}} / 2$. The external field consists of a hard, repulsive background $\left(\varphi_{\mathrm{hs}}\right)$ with a superimposed attractive contribution represented by

$$
\Phi_{\text {ext }}(z, \omega)=\varepsilon^{\prime} \sum_{k=1}^{2} \varphi_{\text {sw }}\left(\Delta z^{(k)}\right) g^{(k)}(\omega),
$$

where $\varphi_{\text {sw }}$ is given in Eq. (2.3) and the dimensionless constant $\varepsilon^{\prime}$ is introduced to allow us to vary the strength of the fluidsubstrate attraction relative to the fluid-fluid one.

The function $g^{(k)}$ appearing on the right-hand side of Eq. (2.7) is the so-called anchoring function. It permits us
TABLE I. Anchoring scenarios [see Eqs. (2.9)-(2.11)]. Entries in the first

\begin{tabular}{|c|c|c|c|c|c|c|c|c|}
\hline \multicolumn{2}{|c|}{ Substrate } & & \multirow[b]{2}{*}{$\alpha^{(1)}$} & \multirow[b]{2}{*}{$\beta^{(1)}$} & \multirow[b]{2}{*}{$\alpha^{(2)}$} & \multirow[b]{2}{*}{$\beta^{(2)}$} & \multirow[b]{2}{*}{$\sqrt{\frac{3}{8 \pi}} g^{(1)}$} & \multirow[b]{2}{*}{$\sqrt{\frac{3}{8 \pi}} g^{(2)}$} \\
\hline$k=1$ & $k=2$ & & & & & & & \\
\hline$+x$ & $+x$ & $p$ & $\frac{\pi}{2}$ & 0 & $\frac{\pi}{2}$ & 0 & $-\operatorname{Re} \mathcal{Y}_{11}$ & $-\operatorname{Re} \mathcal{Y}_{11}$ \\
\hline$+x$ & $+y$ & $o$ & $\frac{\pi}{2}$ & 0 & $\frac{\pi}{2}$ & $\frac{\pi}{2}$ & $-\operatorname{Re} \mathcal{Y}_{11}$ & $-\operatorname{Im} \mathcal{Y}_{11}$ \\
\hline$+x$ & $-x$ & $a_{x}$ & $\frac{\pi}{2}$ & 0 & $\frac{3 \pi}{2}$ & 0 & $-\operatorname{Re} \mathcal{Y}_{11}$ & $+\operatorname{Re} \mathcal{Y}_{11}$ \\
\hline$+z$ & $-z$ & $a_{z}$ & 0 & 0 & $\pi$ & 0 & $+\mathcal{Y}_{10} / \sqrt{2}$ & $-\mathcal{Y}_{10} / \sqrt{2}$ \\
\hline
\end{tabular}
two columns give the direction of the preferred alignment at the lower $(k=1)$ and upper $(k=2)$ substrate. Entries in the third column refer to the anchoring scenario where we restrict the discussion to parallel $(p)$, orthogonal $(o)$, and antiparallel $\left(a_{x}\right)$ preferred relative orientation of molecules at both walls. Notice that for the $a_{z}$ anchoring scenario, the choice of $\beta^{(1)}$ and $\beta^{(2)}$ is arbitrary.

to energetically discriminate certain orientations of a polar molecule with respect to a wall. More specifically,

$$
g^{(k)}(\omega)=\widehat{\boldsymbol{u}}(\omega) \cdot \widehat{\boldsymbol{e}}^{(k)} .
$$

We express $\widehat{\boldsymbol{u}}$ in spherical coordinates according to

$$
\widehat{\boldsymbol{u}}(\omega)=\left(\begin{array}{c}
\sin \theta \cos \phi \\
\sin \theta \sin \phi \\
\cos \theta
\end{array}\right)=\sqrt{\frac{8 \pi}{3}}\left(\begin{array}{l}
-\operatorname{Re} \mathcal{Y}_{11}(\omega) \\
-\operatorname{Im} \mathcal{Y}_{11}(\omega) \\
\mathcal{Y}_{10}(\omega) / \sqrt{2}
\end{array}\right),
$$

where Re and Im refer to real and imaginary parts of $\mathcal{Y}_{11}$, respectively, and Eq. (A.62) of Ref. 29 has also been used. Let now the preferred alignment of molecules at a substrate be given in general by the expression

$$
\widehat{\boldsymbol{e}}^{(k)}=\left(\begin{array}{c}
\sin \alpha^{(k)} \cos \beta^{(k)} \\
\sin \alpha^{(k)} \sin \beta^{(k)} \\
\cos \alpha^{(k)}
\end{array}\right), \quad k=1,2,
$$

where $\alpha^{(k)}$ is the angle between the preferred alignment of a molecule and the $z$-axis and $\beta^{(k)}$ specifies a distinguished direction in the $x-y$ plane with which the molecules should align. We shall focus on three particularly interesting situations compiled in Table I which all relate to orientations in the $x$ $-y$ plane; for the sake of comparison, we also consider one anchoring scenario along the $z$-axis.

\section{THEORY}

\section{A. Thermodynamic integration}

To determine the phase behavior and structure of the confined spin fluid of thermodynamically stable phases, we are seeking minima of the grand potential which are treated as a functional of the orientation-dependent singlet density $\rho .{ }^{29} \mathrm{It}$ can be expressed in general as

$\Omega[\rho(\boldsymbol{r}, \omega)]=\mathcal{F}[\rho(\boldsymbol{r}, \omega)]+\iint \mathrm{d} \boldsymbol{r} \mathrm{d} \omega\left[\Phi_{\text {ext }}(\boldsymbol{r}, \omega)-\mu\right] \rho(\boldsymbol{r}, \omega)$,

where $\mathcal{F}$ is the intrinsic free-energy functional, $\Phi_{\text {ext }}$ is an external potential (see Sec. III B), and $\mu$ is the chemical potential. Hence, the grand-potential functional $\Omega$ can formally be perceived as a generalised Legendre transform of $\mathcal{F}$. 
To arrive at a tractable expression for the free-energy functional in Eq. (3.1), we decompose the total configurational potential energy according to

$$
\Phi\left(\boldsymbol{\Gamma}_{N} ; \xi\right)=\Phi_{0}\left(\boldsymbol{r}^{N}\right)+\xi \Phi_{1}\left(\boldsymbol{\Gamma}_{N}\right),
$$

where $\boldsymbol{\Gamma}_{N} \equiv\left(\boldsymbol{r}^{N}, \omega^{N}\right)$ is a point in the $5 N$-dimensional configuration space spanned by the set of $3 N$ center-of-mass coordinates $\boldsymbol{r}^{N}=\left\{\boldsymbol{r}_{1}, \boldsymbol{r}_{2}, \ldots, \boldsymbol{r}_{N}\right\}$ and the corresponding $2 N$ Euler angles $\omega^{N}=\left\{\omega_{1}, \omega_{2}, \ldots, \omega_{N}\right\}$ specifying the orientations of our uniaxially symmetric molecules.

In Eq. (3.2), $\Phi_{0}$ is the configurational potential energy of an (suitably chosen) isotropic reference system, whereas $\Phi_{1}$ refers to the configurational potential energy of a perturbation that accounts for the orientation dependence; $0 \leq \xi$ $\leq 1$ is a dimensionless coupling parameter introduced to specify a linear path that takes us from a reference system (subscript $0, \xi=0)$ to the system of interest $(\xi=1)$. Notice that both the reference system and the perturbed system are treated in confinement and are thus inhomogeneous and anisotropic despite the spherically symmetric interaction potential $\varphi_{0}$.

We assume that $\Phi_{0}$ and $\Phi_{1}$ can be obtained from pairwise additive potentials $\varphi_{0}$ and $\varphi_{1}$, respectively. In line with the previous work on a related model, ${ }^{30}$ we define $\varphi_{1}$ such that

$$
\begin{aligned}
\left\langle\varphi_{1}\left(\boldsymbol{r}_{12}, \omega_{1}, \omega_{2}\right)\right\rangle_{\omega_{1}, \omega_{2}} & =\frac{1}{(4 \pi)^{2}} \int \mathrm{d} \omega_{1} \mathrm{~d} \omega_{2} \varphi_{1}\left(\boldsymbol{r}_{12}, \omega_{1}, \omega_{2}\right) \\
& =0
\end{aligned}
$$

Owing to the "multipole-like" character ${ }^{31}$ of $\varphi_{\text {anis }}$ that is because of

$$
\int \mathrm{d} \omega_{1} \mathrm{~d} \omega_{2} \Phi_{110}\left(\omega_{1}, \omega_{2}, \omega\right)=0
$$

we realise that $\varphi_{1}=\varphi_{\text {anis }}$. Equation (3.4) is a consequence of the orthogonality of rotational invariants which is demonstrated in Appendix B of Ref. 30.

Because of this choice of $\varphi_{1}$, it immediately follows from Eq. (2.1) that

$\varphi_{0}\left(r_{12}\right)=\varphi_{\mathrm{ff}}\left(\boldsymbol{r}_{12}, \omega_{1}, \omega_{2}\right)-\varphi_{1}\left(\boldsymbol{r}_{12}, \omega_{1}, \omega_{2}\right)=\varphi_{\text {iso }}\left(r_{12}\right)$.

At this stage, we invoke principles of thermodynamic integration as detailed in Ref. 30 which permit us to write

$$
\begin{aligned}
\Delta \mathcal{F}= & \mathcal{F}-\mathcal{F}_{0} \\
= & \frac{1}{2} \int_{0}^{1} \mathrm{~d} \xi \iint \mathrm{d} \boldsymbol{r}_{1} \mathrm{~d} \boldsymbol{r}_{2} \mathrm{~d} \omega_{1} \mathrm{~d} \omega_{2} \varphi_{1}\left(\boldsymbol{r}_{12}, \omega_{1}, \omega_{2}\right) \\
& \times \rho\left(\boldsymbol{r}_{1}, \omega_{1}\right) \rho\left(\boldsymbol{r}_{2}, \omega_{2}\right) g\left(\boldsymbol{r}_{1}, \boldsymbol{r}_{2}, \omega_{1}, \omega_{2} ; \xi\right),
\end{aligned}
$$

where $\mathcal{F}_{0}$ is the free-energy functional associated with $\varphi_{0}$ and $\mathcal{F}$ is the functional of the free energy of the system of interest [in which interactions are described by $\varphi_{\mathrm{ff}}$; see Eq. (2.1)]; $g$ is the orientation-dependent pair correlation function for the inhomogeneous and anisotropic confined fluid that depends on the coupling parameter $\xi$.

\section{B. Free-energy functional of the reference system}

Because in our model $\Phi_{\text {ext }}$ depends only on $z$ [see Eq. (2.8)], properties of the confined fluid are translationally invariant across the $x-y$ plane at a given $z$. Thus, we may simplify the generic singlet distribution function according to

$$
\rho(\boldsymbol{r}, \omega) \rightarrow \rho(z, \omega)=\rho(z) \alpha(z, \omega),
$$

where $\rho$ on the far right-hand side is the local density and $\alpha$ is the orientation distribution function. The latter is normalised according to

$$
\int \mathrm{d} \omega \alpha(z, \omega)=1
$$

An immediate consequence of Eqs. (3.7) and (3.8) is that we can write

$$
\mathcal{F}_{0}[\rho(z, \omega)]=\mathcal{F}_{\text {id }}[\rho(z, \omega)]+\mathcal{F}_{\mathrm{hs}}[\rho(z, \omega)]+\mathcal{F}_{\mathrm{sw}}[\rho(z, \omega)]
$$

for the free energy functional of the inhomogeneous reference fluid where $\mathcal{F}_{\text {id }}$ refers to an ideal-gas contribution, $\mathcal{F}_{\mathrm{hs}}$ accounts for the contribution from the hard-core repulsion, and $\mathcal{F}_{\text {sw }}$ is a contribution from the attractive trough [see Eq. (2.3)].

Starting with the ideal-gas contribution, this can be cast as a sum of two terms, namely,

$$
\begin{aligned}
\frac{\beta \mathcal{F}_{\text {id }}}{A}= & \int \mathrm{d} z \rho(z)\left\{\ln \left[\rho(z) \Lambda^{5} m / I\right]-1\right\} \\
& +\iint \mathrm{d} z \mathrm{~d} \omega \rho(z) \alpha(z, \omega) \ln [4 \pi \alpha(z, \omega)],
\end{aligned}
$$

where $A$ is the area of a substrate and $\beta=1 / k_{\mathrm{B}} T ; k_{\mathrm{B}}$ is Boltzmann's constant and $T$ is temperature. The first term on the right-hand side of Eq. (3.10) accounts for the kineticenergy contribution to $\mathcal{F}_{\text {id }}$. This is evident from the exponent of 5 of the thermal de Broglie wavelength $\Lambda$ because our uniaxially symmetric molecules have three translational and two rotational degrees of freedom; $m$ is the molecular mass and $I$ is the moment of inertia of a molecule. Because we are eventually dealing with systems at thermodynamic equilibrium, specific choices for $\Lambda, m$, and $I$ do not matter.

The second term on the right-hand side of Eq. (3.10) is entropic in nature. It accounts for the loss in orientational entropy if the confined fluid undergoes a phase transition from an isotropic (gas or liquid) phase to an ordered (polar) one. The factor of $4 \pi$ is included to make sure that this entropic term vanishes in the isotropic phase where, on account of Eq. (3.8), $\alpha=1 / 4 \pi$ for uniaxially symmetric molecules.

The second term on the right-hand side of Eq. (3.9) is the hard-sphere contribution. Because the system of interest is inhomogeneous and anisotropic, we need to resort to an FMT treatment to deal with this contribution adequately. This is one of the novel aspects of this work in contrast to Paper I. ${ }^{14}$ We therefore defer a more detailed discussion of some of the key ideas of FMT to Sec. III C. However, before concluding the present section, we need to focus on the last term on the right-hand side of Eq. (3.9) accounting for the free-energy contribution of the attractive square-well potential [see Eq. (2.3)]. Because, on the one hand, the interaction potential $\varphi_{\text {sw }}$ is spherically symmetric but, on the other hand, the confined fluid is inhomogeneous and anisotropic on account of $\Phi_{\text {ext }}$, we adopt a simple mean-field treatment of $\mathcal{F}_{\text {sw }}$ by ignoring intermolecular 
correlations altogether. Under these premises, the final result for $\mathcal{F}_{\text {sw }}$ can be cast as

$$
\frac{\beta \mathcal{F}_{\mathrm{sw}}}{A}=\beta \int \mathrm{d} z_{1} \mathrm{~d} z_{2} \rho\left(z_{1}\right) \rho\left(z_{2}\right) \widetilde{\varphi}_{\mathrm{sw}}\left(\left|z_{1}-z_{2}\right|\right),
$$

where $\widetilde{\varphi}_{\text {sw }}$ is introduced in Appendix A.

Although one might think that employing a simple meanfield approximation to pair correlations in the confined fluid may not at all be good in a quantitative sense, we will demonstrate below that this is not necessarily so for the short-range square-well potential employed in this work. This observation is in line with the recent work by Archer et al. ${ }^{32}$ These authors studied a system of one-dimensional hard rods with an attractive tail potential. Comparing their mean-field DFT results with the exact results, they found a surprisingly good agreement for the direct correlation function and for the static structure factor.

\section{Key principles of fundamental measure theory}

We now turn to a discussion of the contribution of the interaction between the hard cores of the molecules. Because our system is anisotropic and inhomogeneous on account of the external field representing the walls of the slit-pore, we employ FMT. We follow the earlier work by Roth et al. ${ }^{23}$ and employ FMT in their White Bear version (Mark I) (see also Ref. 24).

The White Bear version of FMT is an advancement of Rosenfeld's original FMT as described in two ingenious papers quite some time ago ${ }^{15,16}$ in which he and co-workers aimed at treating multicomponent mixtures of hard spheres of different sizes. We will follow this route and then specialize later to a one-component hard-sphere fluid confined to a cylindrically symmetric slit-pore.

The starting point in the development of FMT is the assumption that in a multicomponent mixture of hard spheres the free-energy functional can be expressed as

$$
\beta \mathcal{F}_{\mathrm{hs}}\left[\left\{\rho_{i}(\boldsymbol{r})\right\}\right]=\int \mathrm{d} \boldsymbol{r} \Phi\left(\left\{n_{\alpha}(\boldsymbol{r})\right\}\right),
$$

where $\Phi$ is a reduced free-energy density that depends on a set of weighted densities $\left\{n_{\alpha}\right\}$. Technically speaking, $\Phi$ is a function of the members of the set $\left\{n_{\alpha}\right\}$, whereas $\mathcal{F}_{\mathrm{hs}}$ is a functional of the set $\left\{\rho_{i}\right\}$. It is important to keep this distinction in mind for the subsequent development of the theory. The weighted densities in Eq. (3.12) can be obtained from the convolution of the set $\left\{\rho_{i}\right\}$,

$$
n_{\alpha}(\boldsymbol{r})=\sum_{i} \int \mathrm{d} \boldsymbol{r}^{\prime} \rho_{i}(\boldsymbol{r}) w_{\alpha}^{i}\left(\left|\boldsymbol{r}-\boldsymbol{r}^{\prime}\right|\right)
$$

with a set of weight functions $\left\{w_{\alpha}^{i}\right\}$ that we shall specify later.

However, we note that the weight functions are associated with certain geometrical measures of the hard spheres. For example, $w_{3}^{i}$ is related to the volume of a hard sphere and $w_{2}^{i}$ is associated with its surface. Therefore, $n_{3}$ obtained from the volume integration in Eq. (3.13) is a dimensionless quantity, whereas $n_{2}$ has dimensions of the inverse length. Other weight functions such as $w_{0}^{i}$ and $w_{1}^{i}$ and the two vector weight functions $\boldsymbol{w}_{1}^{i}$ and $\boldsymbol{w}_{2}^{i}$ lead to weighted densities that have dimensions of inverse volume $\left(n_{0}\right)$ and area $\left(n_{1}\right)$, respectively; the two vector weight functions lead to weighted densities that share the dimension with their scalar counterparts. The different dimensions of members of the set $\left\{n_{\alpha}\right\}$ are important later because they justify the ansatz to be made below for $\Phi$.

Next, we consider the grand-potential functional $\Omega_{\mathrm{hs}}$. It can be expressed as ${ }^{27}$

$$
\begin{aligned}
\Omega_{\mathrm{hs}}\left[\left\{\rho_{i}(\boldsymbol{r})\right\}\right] & =-\int \mathrm{d} \boldsymbol{r} P_{\mathrm{hs}}\left[\left\{\rho_{i}(\boldsymbol{r})\right\}\right] \\
& =\mathcal{F}_{\mathrm{hs}}\left[\left\{\rho_{i}(\boldsymbol{r})\right\}\right]-\sum_{i} \int \mathrm{d} \boldsymbol{r} \rho_{i}(\boldsymbol{r}) \frac{\delta \mathcal{F}_{\mathrm{hs}}}{\delta \rho_{i}},
\end{aligned}
$$

where the expression on the first line of the equation is a generalization of the standard thermodynamic expression $\Omega=-P V$ to inhomogeneous fluids; the expression on the second line can be viewed as a generalized Legendre transform of $\mathcal{F}_{\mathrm{hs}}$. The operator $\delta / \delta \rho_{i}$ indicates a functional derivative. Employing now Eq. (3.12), it is relatively straightforward to realize that Eq. (3.14) can be recast as

$$
\begin{aligned}
\beta \Omega_{\mathrm{hs}}\left[\left\{\rho_{i}(\boldsymbol{r})\right\}\right]= & \int \mathrm{d} \boldsymbol{r} \Phi\left(\left\{n_{\alpha}(\boldsymbol{r})\right\}\right)-\sum_{i} \int \mathrm{d} \boldsymbol{r} \rho_{i}(\boldsymbol{r}) \\
& \times \int \mathrm{d} \boldsymbol{r}^{\prime} \sum_{\alpha} \frac{\partial \Phi\left(\left\{n_{\gamma}\left(\boldsymbol{r}^{\prime}\right)\right\}\right)}{\partial n_{\alpha}\left(\boldsymbol{r}^{\prime}\right)} \frac{\delta n_{\alpha}\left(\boldsymbol{r}^{\prime}\right)}{\delta \rho_{i}(\boldsymbol{r})} .
\end{aligned}
$$

Because of Eq. (3.13), the functional derivative in the previous expression can be evaluated to give

$$
\frac{\delta n_{\alpha}\left(\boldsymbol{r}^{\prime}\right)}{\delta \rho_{i}(\boldsymbol{r})}=w_{\alpha}^{i}\left(\left|\boldsymbol{r}^{\prime}-\boldsymbol{r}\right|\right)
$$

which follows because of

$$
\frac{\delta \rho_{j}\left(\boldsymbol{r}^{\prime \prime}\right)}{\delta \rho_{i}\left(\boldsymbol{r}^{\prime}\right)}=\delta\left(\boldsymbol{r}^{\prime}-\boldsymbol{r}^{\prime \prime}\right)
$$

Thus, we realize from Eq. (3.14) that

$$
\beta P_{\mathrm{hs}}\left[\left\{\rho_{i}(\boldsymbol{r})\right\}\right]=-\Phi\left(\left\{n_{\alpha}(\boldsymbol{r})\right\}\right)+\sum_{\alpha} n_{\alpha}(\boldsymbol{r}) \frac{\partial \Phi\left(\left\{n_{\gamma}(\boldsymbol{r})\right\}\right)}{\partial n_{\alpha}(\boldsymbol{r})}
$$

Following the original arguments of Rosenfeld ${ }^{15}$ (see also Ref. 24), one now makes an ansatz for the reduced free-energy density, namely,

$$
\begin{aligned}
\Phi\left(\left\{n_{\alpha}(\boldsymbol{r})\right\}\right)= & f_{1}\left(n_{3}\right) n_{0}+f_{2}\left(n_{3}\right)\left(n_{1} n_{2}-\boldsymbol{n}_{1} \cdot \boldsymbol{n}_{2}\right) \\
& +f_{4}\left(n_{3}\right)\left(n_{2}^{3}-3 n_{2} \boldsymbol{n}_{2} \cdot \boldsymbol{n}_{2}\right),
\end{aligned}
$$

where $f_{1}, f_{2}$, and $f_{4}$ are expansion coefficients. Notice that in general the weighted densities in Eq. (3.19) are nonlocal quantities because of their definition given in Eq. (3.13). However, we have dropped their argument $\boldsymbol{r}$ to ease the notational burden. In addition, this expansion takes notice of the fact that a general expansion involves two more expansion coefficients $f_{3}$ and $f_{5}$ which can be shown to be linearly dependent on $f_{2}$ and $f_{4}$, respectively. ${ }^{24}$

Because the left-hand side of Eq. (3.19) has dimensions of density and because of the dimensionality argument given below Eq. (3.13), only the combinations of weighted densities on the right-hand side of Eq. (3.19) can occur; because $n_{3}$ is 
dimensionless, the expansion coefficients $f_{1}, f_{2}$, and $f_{4}$ can at most depend on $n_{3}$.

Let us now specialize to a homogeneous bulk phase. In this case, terms proportional to the weighted densities $\boldsymbol{n}_{1}$ and $\boldsymbol{n}_{2}$ in Eq. (3.19) vanish because $\int \mathrm{d} \boldsymbol{r} \boldsymbol{w}_{\alpha}^{i}\left(\left|\boldsymbol{r}-\boldsymbol{r}^{\prime}\right|\right)=0$. Moreover, in a homogeneous bulk fluid, the ideal-gas contribution to the pressure $P$ is given by $\beta P_{\mathrm{id}}=\sum_{i} \rho_{i}=n_{0}$. Thus, $P=P_{\mathrm{id}}+P_{\mathrm{hs}}$ and therefore [see Eq. (3.18)]

$$
\beta P=-\Phi\left[\left\{n_{\alpha}\right\}\right]+\sum_{\alpha=0}^{3} n_{\alpha} \frac{\partial \Phi}{\partial n_{\alpha}}+n_{0}
$$

The White-Bear version (Mark I) of FMT ${ }^{23,24}$ then assumes that the left-hand side of the previous expression is given by the Mansoori-Carnahan-Starling-Leeland equation of state, ${ }^{25}$ that is,

$$
\beta P=\frac{n_{0}}{1-n_{3}}+\frac{n_{1} n_{2}}{\left(1-n_{3}\right)^{2}}+\frac{n_{2}^{3}}{12 \pi\left(1-n_{3}\right)^{3}}-\frac{n_{3} n_{2}^{3}}{36 \pi\left(1-n_{3}\right)^{3}} .
$$

Inserting Eq. (3.21) together with the ansatz presented in Eq. (3.19) into Eq. (3.20) gives us three simple differential equations for the expansion coefficients $f_{1}, f_{2}$, and $f_{4}$ in Eq. (3.19). Thus, with the solutions of these differential equations, we can recast Eq. (3.19) as

$$
\begin{aligned}
\Phi\left(\left\{n_{\alpha}(\boldsymbol{r})\right\}\right)= & -n_{0} \ln \left(1-n_{3}\right)+\frac{n_{1} n_{2}-\boldsymbol{n}_{1} \cdot \boldsymbol{n}_{2}}{1-n_{3}} \\
& +\left(n_{2}^{3}-3 n_{2} \boldsymbol{n}_{2} \cdot \boldsymbol{n}_{2}\right) \\
& \times \frac{n_{3}+\left(1-n_{3}\right)^{2} \ln \left(1-n_{3}\right)}{36 \pi\left(1-n_{3}\right)^{2} n_{3}^{2}} .
\end{aligned}
$$

The development of FMT thus far is based upon the assumption of a general inhomogeneous, anisotropic hardsphere fluid multicomponent mixture reflected by the dependence of the weighted densities on the vector position $\boldsymbol{r}$ in Eq. (3.13). However, as indicated by Eq. (2.8), the external potential representing the structureless, planar substrates depends only on the distance of the center of mass of a molecule from the substrate plane. This implies that the weight functions can be integrated over the $x$ - and $y$-coordinates. Moreover, we now specialize to a single-component hardsphere fluid consequently dropping the index $i$ of members of the set of weight functions $\left\{w_{\alpha}\right\}$. Following the previous work, ${ }^{12,24,33}$ the resulting weight functions are then given by the expressions

$$
\begin{aligned}
& w_{0}(z)=\frac{1}{\sigma} \Theta\left(\frac{\sigma}{2}-|z|\right), \\
& w_{1}(z)=\frac{1}{2} \Theta\left(\frac{\sigma}{2}-|z|\right), \\
& w_{2}(z)=\pi \sigma \Theta\left(\frac{\sigma}{2}-|z|\right), \\
& w_{3}(z)=\left[\left(\frac{\sigma}{2}\right)^{2}-z^{2}\right] \Theta\left(\frac{\sigma}{2}-|z|\right), \\
& \boldsymbol{w}_{1}(z)=\frac{z}{\sigma} \widehat{\boldsymbol{e}}_{\mathrm{Z}} \Theta\left(\frac{\sigma}{2}-|z|\right), \\
& \boldsymbol{w}_{2}(z)=2 \pi z \widehat{\boldsymbol{e}}_{\mathrm{Z}} \Theta\left(\frac{\sigma}{2}-|z|\right),
\end{aligned}
$$

where $\widehat{\boldsymbol{e}}_{\mathrm{z}}$ is a unit vector pointing along the $z$-axis of the (Cartesian) coordinate system.

\section{Free-energy functional accounting for anisotropic attractions}

Last but not least, we now need to develop an approximate free energy functional that accounts for the contribution of the anisotropic perturbation potential $\varphi_{1}$. Because of the success of describing the free energy of the square-well reference fluid by a mean-field treatment, we base the following considerations also on a mean-field approximation setting the pair correlation function $g$ in Eq. (3.6) equal to one. We then transform variables in Eq. (3.6) according to $\boldsymbol{r}_{1} \rightarrow \boldsymbol{r}_{1}^{\prime}=\boldsymbol{r}_{1}, \boldsymbol{r}_{2}$ $\rightarrow \boldsymbol{r}_{2}^{\prime}=\boldsymbol{r}_{12}$ and switch to cylindrical coordinates. This permits us to perform the two trivial integrations over $\mathrm{d} \xi$ and over the surface element $\mathrm{d} A_{1}^{\prime}=a_{1}^{\prime} \mathrm{d} a_{1}^{\prime} \mathrm{d} \phi_{1}^{\prime}$ analytically. Thus, we can eventually rewrite Eq. (3.6) for the specific system under consideration as

$$
\begin{aligned}
\frac{\beta \Delta \mathcal{F}}{A}= & -\frac{\beta \varepsilon_{\mathrm{H}}}{2} \frac{(4 \pi)^{3 / 2}}{\sqrt{3}} \int_{0}^{2 \pi} \mathrm{d} \gamma_{12} \int \mathrm{d} z_{1} \mathrm{~d} z_{2} \rho\left(z_{1}\right) \rho\left(z_{2}\right) \\
& \times \int \mathrm{d} \omega_{1} \mathrm{~d} \omega_{2} \alpha\left(z_{1}, \omega_{1}\right) \alpha\left(z_{2}, \omega_{2}\right) \Phi_{110}\left(\omega_{1}, \omega_{2}, \omega\right) \\
& \times \int \mathrm{d} a_{12} a_{12} \varphi_{\mathrm{sw}}\left(r_{12}\right)
\end{aligned}
$$

where $\gamma_{12}$ is the azimuth of the orientation of $\widehat{\boldsymbol{r}}_{12} ; \varphi_{1}=\varphi_{\text {anis }}$ (see discussion in Sec. III A) from Eq. (2.4) and the decomposition of the singlet generic distribution function [see Eq. (3.7)] has also been employed. The integration over $\mathrm{d} \gamma_{12}$ can immediately be carried out and gives a factor of $2 \pi$. This is because, the last index of the rotational invariant is zero, reflecting the fact that the perturbation potential does not depend on $\widehat{\boldsymbol{r}}_{12}$. Notice also that the expression given in Eq. (3.24) implies that $\Delta \mathcal{F}$ is a functional of both the local density and the local orientation distribution function.

We now expand the latter according to

$$
\alpha(z, \omega)=\sum_{L M} \alpha_{L M}(z) \mathcal{Y}_{L M}(\omega)
$$

where members of the sets $\left\{\alpha_{L M}\right\}$ and $\left\{\mathcal{Y}_{L M}\right\}$ are (local) expansion coefficients and spherical harmonics [see Eq. (A.62) of Ref. 29], respectively; $L \geq 0$ and $-L \leq M \leq L$ are integers defined such that for each $L$ the sum over $M$ in Eq. (3.25) involves $2 L+1$ terms. Because the spherical harmonics form a complete set of orthonormal basis functions, Eq. (3.25) can be solved for members of the set $\left\{\alpha_{L M}\right\}$ such that

$$
\alpha_{L M}(z)=\int \mathrm{d} \omega \alpha(z, \omega) \mathcal{Y}_{L M}^{*}(\omega)
$$

Using Eq. (3.25), the integration over orientations in Eq. (3.24) can be performed analytically as we demonstrate in the Appendix of Paper I. ${ }^{14}$ We finally arrive at

$$
\begin{aligned}
\frac{\beta \Delta \mathcal{F}}{A}= & -\frac{4 \pi}{3} \beta \varepsilon_{\mathrm{H}} \int \mathrm{d} z_{1} \mathrm{~d} z_{2} \rho\left(z_{1}\right) \rho\left(z_{2}\right) \widetilde{\varphi}_{\mathrm{sw}}\left(\left|z_{1}-z_{2}\right|\right) \\
& \times \sum_{m=-1}^{1}(-1)^{m} \alpha_{1 \underline{m}}\left(z_{1}\right) \alpha_{1 m}\left(z_{2}\right),
\end{aligned}
$$


where $\widetilde{\varphi}_{\mathrm{sw}}$ is introduced in Eq. (A5) and the notation $\underline{m}=-m^{29}$ has also been used.

\section{THERMODYNAMIC STABILITY}

Henceforth, we shall be focusing on thermodynamically stable states satisfying the conditions

$$
\begin{aligned}
& \frac{\delta \Omega[\rho(z), \alpha(z, \omega)]}{\delta \rho(z)}=0, \\
& \frac{\delta \Omega[\rho(z), \alpha(z, \omega)]}{\delta \alpha(z, \omega)}=\chi[\rho(z), T],
\end{aligned}
$$

where $\chi$ is a Lagrangian multiplier introduced to ensure that the orientation distribution function obtained from Eq. (4.1b) automatically satisfies the constraint given by Eq. (3.8). Because of the discussion in Sec. III, $\Omega$ is a functional of both the local density and the local orientation distribution function and may be cast more explicitly as

$$
\Omega=\mathcal{F}_{0}+\Delta \mathcal{F}+A \iint \mathrm{d} z \mathrm{~d} \omega \rho(z) \alpha(z, \omega)\left[\Phi_{\text {ext }}(z, \omega)-\mu\right],
$$

where $\mathcal{F}_{0}$ [see Eq. (3.9)] and $\Delta \mathcal{F}$ [see Eq. (3.27)] are functionals of the local density and the local orientation distribution functions as well; the external potential $\Phi_{\text {ext }}$ is defined in Eq. (2.8).

From Eq. (4.1a), it follows that

$$
\begin{aligned}
0= & \ln [\rho(z)]+\int \mathrm{d} \omega \alpha(z, \omega) \ln [4 \pi \alpha(z, \omega)] \\
& +\beta \mu_{\mathrm{hs}}[\rho(z)]+2 \beta \int \mathrm{d} z^{\prime} \rho\left(z^{\prime}\right) \widetilde{\varphi}_{\mathrm{sw}}\left(\left|z-z^{\prime}\right|\right) \\
& -\frac{8 \pi}{3} \beta \varepsilon_{\mathrm{H}} \sum_{m=-1}^{1}(-1)^{m} \alpha_{1 \underline{m}}(z) \int \mathrm{d} z^{\prime} \alpha_{l m}\left(z^{\prime}\right) \rho\left(z^{\prime}\right) \\
& \times \widetilde{\varphi}_{\mathrm{sw}}\left(\left|z-z^{\prime}\right|\right)+\beta \int \mathrm{d} \omega \alpha(z, \omega) \Phi_{\mathrm{ext}}(z, \omega)-\beta \mu^{*},
\end{aligned}
$$

where $\mu_{\mathrm{hs}}$ is the hard-sphere chemical potential and $\beta \mu^{*} \equiv \beta \mu-\ln \left(\Lambda^{5} m / I\right)$. Following the work of Szalai and Dietrich $^{12}$ [see also Eqs. (3.12) and (3.13)] expressions for $\mu_{\mathrm{hs}}$ can be obtained from

$$
\begin{aligned}
\beta \mu_{\mathrm{hs}}[\rho(z)] & \equiv \beta \frac{\delta \mathcal{F}_{\mathrm{hs}}[\rho(z)]}{\delta \rho(z)} \\
& =\sum_{\alpha=0}^{5} \int \mathrm{d} z \frac{\partial \Phi\left(\left\{n_{\alpha}(z)\right\}\right)}{\partial n_{\alpha}(z)} w_{\alpha}\left(\left|z-z^{\prime}\right|\right),
\end{aligned}
$$

where the weight functions are given in Eqs. (3.23); in particular, expressions for $\alpha=4,5$ refer to the vector weight functions $\boldsymbol{w}_{1}$ and $\boldsymbol{w}_{2}$ given in Eqs. (3.23e) and (3.23f), respectively. The final expressions for the various partial derivatives of $\Phi$ in Eq. (4.4) can be found in the paper by Szalai and Dietrich where a sign error occurs in the expression for $\partial \Phi / \partial n_{3} .^{12}$

We can simplify Eq. (4.3) by eliminating the second term on the right-hand side. To that end, we first replace the expansion coefficients using Eq. (3.26) for $L=1$ and invoke Eq. (A.27) of Ref. 29. Next, we take the functional derivative of the resulting expression with respect to $\alpha$ which then gives [see Eq. (4.1b)]

$$
\begin{aligned}
\frac{\chi[\rho(z), T]-\rho(z)}{\rho(z)}= & \ln [4 \pi \alpha(z, \omega)]+\beta \Phi_{\mathrm{ext}}(z, \omega) \\
& -\frac{8 \pi}{3} \beta \varepsilon_{\mathrm{H}} \sum_{m=-1}^{1}(-1)^{m} \mathcal{Y}_{1 m}^{*}(\omega) \\
& \times \int \mathrm{d} z^{\prime} \rho\left(z^{\prime}\right) \alpha_{1 \underline{m}}\left(z^{\prime}\right) \widetilde{\varphi}_{\mathrm{sw}}\left(\left|z-z^{\prime}\right|\right),
\end{aligned}
$$

which can be solved for the orientation distribution function, that is,

$$
\alpha(z, \omega)=\frac{1}{4 \pi} \exp \left\{\frac{\chi[\rho(z), T]-\rho(z)}{\rho(z)}\right\} \Psi(z, \omega),
$$

where

$$
\begin{aligned}
\Psi(z, \omega) \equiv & \exp \left\{-\beta\left[\Phi_{\mathrm{ext}}(z, \omega)\right.\right. \\
& +\frac{8 \pi}{3} \varepsilon_{\mathrm{H}} \sum_{m=-1}^{1}(-1)^{m} \mathcal{Y}_{1 m}^{*}(\omega) \\
& \left.\left.\times \int \mathrm{d} z^{\prime} \rho\left(z^{\prime}\right) \alpha_{1 \underline{m}}\left(z^{\prime}\right) \widetilde{\varphi}_{\mathrm{sw}}\left(\left|z-z^{\prime}\right|\right)\right]\right\} .
\end{aligned}
$$

From this expression and keeping in mind that $\alpha$ is normalized [see Eq. (3.8)], it is straightforward to derive an expression for $\alpha \ln (4 \pi \alpha)$. Replacing with this expression the corresponding term in Eq. (4.3) then yields

$$
\begin{aligned}
0= & \ln \rho(z)+\beta \mu_{\mathrm{hs}}[\rho(z)]-\ln \langle\Psi(z, \omega)\rangle_{\omega}-\beta \mu^{*} \\
& +2 \beta \int \mathrm{d} z^{\prime} \rho\left(z^{\prime}\right) \widetilde{\varphi}_{\mathrm{sw}}\left(\left|z-z^{\prime}\right|\right),
\end{aligned}
$$

where $\langle\ldots\rangle_{\omega} \equiv(1 / 4 \pi) \int \mathrm{d} \omega \ldots$ is an unweighted average over orientations. Solving Eq. (4.8) for $\rho$, we can rewrite it as

$$
\begin{aligned}
\rho(z)= & \exp \left\{-\left[\beta \mu_{\mathrm{hs}}[\rho(z)]-\ln \langle\Psi(z, \omega)\rangle_{\omega}-\beta \mu^{*}\right.\right. \\
& \left.\left.+2 \beta \int \mathrm{d} z^{\prime} \rho\left(z^{\prime}\right) \widetilde{\varphi}_{\mathrm{sw}}\left(\left|z-z^{\prime}\right|\right)\right]\right\},
\end{aligned}
$$

which is amenable to the Picard iteration scheme, ${ }^{34}$ provided that suitable initial values for $\rho$ and $\Psi$ can be provided. The latter can be obtained by realizing from Eqs. (3.26) $(L=1)$, (4.6), and (4.7) solutions of the equation

$$
0=\alpha_{1 m}(z)-\frac{\int \mathrm{d} \omega \mathcal{Y}_{1 m}^{*}(\omega) \Psi(z, \omega)}{\int \mathrm{d} \omega \Psi(z, \omega)}
$$

correspond to (globally or meta-) stable thermodynamic states. Equation (4.10) provides us with three additional equations (because of the relation $-1 \leq m \leq 1$ ) that need to be solved iteratively and simultaneously together with Eq. (4.9).

Depending on the thermodynamic state, the anisotropic square-well fluid introduced in Sec. II is capable of forming isotropic or polar liquid phases. As the orientation distribution function is constant in the isotropic phase, we conclude from Eqs. (3.26) and (A.38) of Ref. 29 that in the isotropic phase members of the set $\left\{\alpha_{1 m}\right\}$ vanish.

Whether or not an isotropic liquid phase forms and under what conditions it may be thermodynamically stable depend 
on the nature of $\Phi_{\text {ext }}$. To realize this, let us rewrite Eq. (3.6) as

$$
\mathcal{F}=\mathcal{F}_{0}+\Delta \mathcal{F}=\mathcal{F}_{\text {or }}+\mathcal{F}_{\text {nor }},
$$

where $\mathcal{F}_{\text {nor }}$ accounts for all contributions to the free energy that do not depend on the orientation of the molecules [see the first term on the right-hand side of Eqs. (3.10)-(3.12)]; the orientation-dependent contributions can then be grouped together according to

$$
\begin{aligned}
\frac{\beta \mathcal{F}_{\text {or }}}{A}= & -\frac{4 \pi}{3} \beta \varepsilon_{\mathrm{H}} \int \mathrm{d} z_{1} \mathrm{~d} z_{2} \rho\left(z_{1}\right) \rho\left(z_{2}\right) \widetilde{\varphi}_{\mathrm{sw}}\left(\left|z_{1}-z_{2}\right|\right) \\
& \times \sum_{m=-1}^{1}(-1)^{m} \alpha_{1 \underline{m}}\left(z_{1}\right) \alpha_{1 m}\left(z_{2}\right) \\
& +\iint \mathrm{d} z \mathrm{~d} \omega \rho(z)\left[\frac{1}{4 \pi}+\xi(z, \omega)\right] \ln [1+4 \pi \xi(z, \omega)] \\
& +\frac{\beta \mathcal{F}_{\text {ext }}}{A},
\end{aligned}
$$

where

$$
\xi(z, \omega) \equiv \sum_{m=-1}^{1} \alpha_{1 m}(z) \mathcal{Y}_{1 m}(\omega)
$$

and Eqs. (3.10), (3.11), and (3.27) and [cf., Eq. (4.2)]

$$
\frac{\beta \mathcal{F}_{\text {ext }}}{A}=\beta \iint \mathrm{d} z \mathrm{~d} \omega \rho(z) \alpha(z, \omega) \Phi_{\text {ext }}(z, \omega)
$$

have also been employed.

We can simplify the expression given in Eq. (4.12) further by realizing that if the system is very weakly polar locally, $\xi$ $\approx 0$. We can then expand the logarithmic term in Eq. (4.12) in a power series in terms of $\xi$. Retaining in this series only the leading terms, it is straightforward to verify that $\ln (1+4 \pi \xi)$ $=\xi-2 \pi \xi^{2}+\mathcal{O}\left(\xi^{3}\right)$.

Using this expansion and Eq. (A.38) of Ref. 29, it is easy to see that the integral over $\mathrm{d} \omega$ in Eq. (4.12) vanishes if the integrand is proportional to $\xi$; because of Eq. (A.39) of Ref. 29, the integral over $\mathrm{d} \omega$ gives $(-1)^{m} \delta_{m \underline{m}}$ if the integrand in Eq. (4.12) contains $\xi$ quadratically. Here, $\delta_{m \underline{m}}$ denotes the Kronecker symbol. Hence, with this simplification, Eq. (4.12) can be rewritten more compactly as

$$
\begin{aligned}
\frac{\beta \mathcal{F}_{\text {or }}}{A}= & -\frac{4 \pi}{3} \beta \varepsilon_{\mathrm{H}} \int \mathrm{d} z_{1} \mathrm{~d} z_{2} \rho\left(z_{1}\right) \rho\left(z_{2}\right) \widetilde{\varphi}_{\mathrm{sw}}\left(\left|z_{1}-z_{2}\right|\right) \\
& \times \sum_{m=-1}^{1}(-1)^{m} \alpha_{1 \underline{m}}\left(z_{1}\right) \alpha_{1 m}\left(z_{2}\right) \\
& +2 \pi \sum_{m=-1}^{1}(-1)^{m} \int \mathrm{d} z \rho(z) \alpha_{1 \underline{m}}(z) \alpha_{1 m}(z)+\frac{\beta \mathcal{F}_{\text {ext }}}{A} .
\end{aligned}
$$

Let us now consider the most general case where the walls can impose polar order in the confined fluid in a small volume $A \Delta z^{(k)}$ near wall $k$. In other words, $\Phi_{\text {ext }}$ in Eq. (2.8) is a function of both $z$ and $\omega$ [through the anchoring function; see Eqs. (2.9)(2.11)]. One can then see from Eq. (4.7) and the consistency relation Eq. (4.10) that it is impossible to have $\alpha_{1 m}=0$ in this so defined volume. Thus, defining as a globally isotropic phase, one in which $\left|\bar{\alpha}_{1 m}\right|=s_{\mathrm{Z}}^{-1} \int \mathrm{d} z\left|\alpha_{1 m}(z)\right|$ vanishes (regardless of $m$ ), it is clear that the presence of an orientation dependent external field prevents an isotropic phase to ever become thermodynamically stable because there will always be a residual nonzero $\left|\bar{\alpha}_{1 m}\right|$ all the way to the critical point.

Next consider a case in which $\Phi_{\text {ext }}$ does not depend on $\omega$. It is then clear from Eqs. (4.7) that the term proportional to $\exp \left(-\beta \Phi_{\text {ext }}\right)$ cancels between the numerator and denominator in the consistency relation in Eq. (4.10) because it can be pulled out from both integrands on the right-hand side. In this case, solutions of Eq. (4.10) are conceivable in which all members of the set $\left\{\alpha_{1 m}\right\}$ are either zero (isotropic phase) or (some or all of these variables) are nonzero (polar phase). Notice also that the term $\beta \mathcal{F}_{\text {ext }} / A$ in Eq. (4.15) is the same in the isotropic and polar phases.

To locate the thermodynamic state at which the polar phase becomes thermodynamically unstable, all terms that depend explicitly on the set $\left\{\alpha_{1 m}\right\}$ have to vanish. To that end and because phase diagrams are usually constructed through the pore-averaged quantities, we introduce $\bar{\rho} \equiv s_{\mathrm{z}}^{-1} \int \mathrm{d} z \rho(z)$. Replacing in Eq. (4.15) all local quantities by their poreaveraged counterparts allows us to estimate the conditions under which the polar phase becomes unstable. We can then transform variables in the first integral on the right-hand side of Eq. (4.15) according to $z_{1} \rightarrow z_{1}^{\prime}=z_{1}$ and $z_{2} \rightarrow z_{12}=\left|z_{1}-z_{2}\right|$ and perform the one trivial integration over $\mathrm{d} z_{1}^{\prime}$ analytically to get

$\frac{\beta \mathcal{F}_{\mathrm{or}}}{V}=\bar{\rho} \sum_{m=-1}^{1}(-1)^{m} \bar{\alpha}_{1 \underline{m}} \bar{\alpha}_{1 m}[u(\beta) \bar{\rho}-2 \pi]+\frac{\beta \mathcal{F}_{\mathrm{ext}}}{V}$,

where the temperature dependent energy parameter is given by

$$
u(\beta)=-\frac{4 \pi}{3} \beta \varepsilon_{\mathrm{H}} \int \mathrm{d} z_{12} \widetilde{\varphi}_{\mathrm{sw}}\left(z_{12}\right) .
$$

The polar phase eventually becomes unstable if the first term on the right-hand side of the previous expression vanishes. Disregarding the trivial cases $\bar{\rho}=0$ and $\bar{\alpha}_{1 m}=0$, another possibility is that the term in brackets vanishes. This allows us to conclude that there is a line of critical points described by the expression $\bar{\rho}=2 \pi / u(\beta)$; the critical line ends at the cep at which the critical line joins the remainder of the phase diagram. The expression for the critical line, which is the analog of the Curie line in ferroelectrics, is wellknown for the Heisenberg fluid ${ }^{35}$ and for dipolar fluids ${ }^{1,4}$ in the bulk.

\section{RESULTS}

\section{A. Polarization}

Our main focus in this study is on the interplay between layering in confined fluids and orientational order. Depending on the specific anchoring scenarios compiled in Table I, we need to quantify both the degree of orientational order and the direction with which the molecules align on average. As in Paper $\mathrm{I}^{14}$ (and as suggested earlier by Gramzow and $\mathrm{Klapp}^{11}$ ), both issues can be addressed by computing the local polarization

$$
\boldsymbol{P}(z)=\int \mathrm{d} \omega \alpha(z, \omega) \widehat{\boldsymbol{u}}(\omega)
$$


where $\widehat{\boldsymbol{u}}$ is given in Eq. (2.10). From this latter equation, it follows that components of $\boldsymbol{P}$ are given by

$$
\begin{aligned}
P_{\mathrm{X}}(z) & =\int \mathrm{d} \omega \alpha(z, \omega) \sin \theta \cos \phi \\
& =-\sqrt{\frac{4 \pi}{3}}\left(\frac{1}{2}\right)^{1 / 2} \int \mathrm{d} \omega \alpha(z, \omega)\left[\mathcal{Y}_{11}(\omega)+\mathcal{Y}_{11}^{*}(\omega)\right] \\
& =-\sqrt{\frac{8 \pi}{3}} \operatorname{Re} \alpha_{11}(z),
\end{aligned}
$$

where Eqs. (A.62) and (A.63) of Ref. 29 as well as Eq. (3.26) have been employed. In a similar fashion, one can show that

$$
\begin{aligned}
& P_{\mathrm{y}}(z)=\sqrt{\frac{8 \pi}{3}} \operatorname{Im} \alpha_{11}(z), \\
& P_{\mathrm{z}}(z)=\sqrt{\frac{4 \pi}{3}} \alpha_{10}(z) .
\end{aligned}
$$

The direction of polarization is then given by the (local) unit vector $\widehat{\boldsymbol{P}}=\boldsymbol{P} / P$ where $P=|\boldsymbol{P}|$.

At this stage, we remind ourselves that the results presented below are based upon a simultaneous solution of Eqs. (4.9) and (4.10). These two equations require at their cores the (unnormalized) orientation distribution function $\Psi$ given in Eq. (4.7).

It depends, on the one hand, on the external field $\Phi_{\text {ext }}$ introduced in Eq. (2.8). Let us consider antiparallel anchoring with the $\pm x$-direction (see Table I) and assume that a particle interacting with one or the other wall is fully aligned with the anchoring direction. In both the cases, it is easy to verify from Eq. (2.9) that $g^{(1)}=g^{(2)}=1$. The same would be true for the $a_{z}$ anchoring scenario if the molecules are perfectly aligned with the $\pm z$-directions (see last entry in Table I) so that over the range $\Delta z^{(k)}<\lambda \sigma$ and for perfectly aligned molecules $\Phi_{\text {ext }}$ would be the same for both the $a_{x}$ and $a_{z}$ anchoring scenarios.

On the other hand, the expression for $\Psi$ in Eq. (4.7) involves

$$
\sum_{m=-1}^{1}(-1)^{m} \mathcal{Y}_{1 m}^{*}(\omega) \alpha_{1 \underline{m}}\left(z^{\prime}\right)=\frac{3}{4 \pi} \widehat{\boldsymbol{u}}(\omega) \cdot \boldsymbol{P}\left(z^{\prime}\right),
$$

where Eqs. (2.10) and (5.2)-(5.4) have also been used. Moreover, we introduce

$$
\widehat{\boldsymbol{u}}(\omega) \cdot \boldsymbol{P}\left(z^{\prime}\right)=\cos \left[\gamma\left(z^{\prime} ; \omega\right)\right] P\left(z^{\prime}\right),
$$

where $\gamma$ is the angle between $\widehat{\boldsymbol{u}}$ and $\boldsymbol{P}$. Hence, with Eqs. (5.5) and (5.6), we can eventually rewrite Eq. (4.7) as

$$
\begin{aligned}
\Psi(z, \omega)= & \exp \left\{-\beta\left[\Phi_{\text {ext }}(z, \omega)\right.\right. \\
& +2 \varepsilon_{\mathrm{H}} \int \mathrm{d} z^{\prime} \rho\left(z^{\prime}\right) \cos \left[\gamma\left(z^{\prime} ; \omega\right)\right] P\left(z^{\prime}\right) \\
& \left.\left.\times \widetilde{\varphi}_{\mathrm{sw}}\left(\left|z-z^{\prime}\right|\right)\right]\right\} .
\end{aligned}
$$

A couple of comments seem appropriate at this point. First, we realize from Eqs. (2.8)-(2.11) that it is energetically most favorable if the molecules align perfectly with $\widehat{\boldsymbol{e}}^{(k)}$ over the range $\Delta z^{(k)}<\lambda \sigma$. Likewise, if under these conditions, $\left|z-z^{\prime}\right|$ is also smaller than $\lambda \sigma$, the integrand in Eq. (5.7) becomes energetically most favorable if $\gamma=0$. From this, it follows that the anchoring conditions determine the direction of $\boldsymbol{P}$. Second, the magnitude $P$ itself is independent of $\widehat{\boldsymbol{e}}^{(k)}$, which prompts us to conclude that across the pore we expect identical polarization profiles where only the component of $\boldsymbol{P}$ differs between different anchoring scenarios $a_{x}$ and $a_{z}$.

\section{B. Numerical details}

From Eqs. (5.2)-(5.4), it is evident that a computation of $\boldsymbol{P}$ requires at its core the set of expansion coefficients $\left\{\alpha_{1 m}\right\}$, which is, in principle, accessible via Eq. (4.10). However, it is clear from considerations in Sec. IV that this protocol boils down to a threefold integration over $z^{\prime}$ [see Eq. (4.7)] and the angles $\theta$ and $\phi$ (i.e., the set of Euler angles $\omega$ ) [see Eq. (4.10)] which is computationally quite demanding (see discussion in the subsequent paragraph). In some cases, we are also interested in the pore average polarization defined through the expression $\bar{P} \equiv s_{\mathrm{z}}^{-1} \int \mathrm{d} z P(z)$.

Fortunately, the integration over angles can be simplified considerably if one uses modified Bessel functions of the first kind. ${ }^{26}$ This is because, these functions have both an integral and a series representation such that one of the integrations over angles can be replaced by an evaluation of the respective series (see Appendix B). Luckily, the latter converges quite rapidly in practice.

For example, if a twofold integration over angles is implemented to solve Eq. (4.10) numerically with a mesh width of $\delta \theta=\delta \phi=10^{-2}$, a CPU time of about $2.8 \times 10^{-2} \mathrm{~s}$ is required to compute all four integrals in Eqs. (B1a)-(B1d); this time increases to about $173 \mathrm{~s}$ if the mesh width is reduced to $\delta \theta=\delta \phi=10^{-4}$. Compared with this latter case, identical results are obtained by the approach involving modified Bessel functions where for the series representation $m \approx 10$ in Eqs. (B12), (B13c)-(B14b) is used; in this latter case, however, the required CPU time is only $6.0 \times 10^{-4} \mathrm{~s}$ which corresponds to a speedup of more than five orders of magnitude. These numbers refer to the computationally most demanding situation discussed in Appendix B as in case 4 in which all three components of $\boldsymbol{P}$ are nonzero.

In practice and depending on the anchoring scenario chosen, four different situations can arise. In the simplest of these, the confined fluid is locally isotropic (case 1 in Appendix B). Noting that with the definitions given in Eqs. (B1a)-(B1d), $\alpha_{10}=\mathcal{J}_{1} / \mathcal{J}_{0}=0, \operatorname{Re} \alpha_{11}=\mathcal{J}_{2} / \mathcal{J}_{0}=0$, and $\operatorname{Im} \alpha_{11}$ $=\mathcal{J}_{3} / \mathcal{J}_{0}=0$, where we used specific values for the four integrals discussed in Appendix B. Thus, from Eqs. (5.2)-(5.4), it follows that $\boldsymbol{P}=\mathbf{0}$ in Eq. (5.1). By a similar token, it follows that in cases 2 and $3 P_{\mathrm{x}}$ and $P_{\mathrm{y}}$ are nonzero but $P_{\mathrm{z}}=0$ and that $P_{\mathrm{x}}=P_{\mathrm{y}}=0$ but $P_{\mathrm{z}} \neq 0$, respectively. Only in case 4 we would find nonzero components $P_{\mathrm{x}}, P_{\mathrm{y}}$, and $P_{\mathrm{z}}$.

In addition to polarization profiles in ordered phases, we are also interested in the global phase behavior of the confined spin fluids. Noting from phenomenological thermodynamics that

$$
\left(\frac{\partial(\Omega / V)}{\partial \mu}\right)_{V, T}=-\bar{\rho}
$$

we can construct plots of $\bar{\rho}$ versus $\mu$ along a sufficiently subcritical isotherm including at either substrate the excluded volume 
$A \sigma / 2$ where the local density vanishes on account of the hard repulsive fluid-substrate interaction. Hence, as $s_{\mathrm{Z}} \rightarrow \infty$, the pore-averaged density approaches its bulk value from below.

In the following, we shall express all physical quantities in appropriate dimensionless (i.e., "reduced") units. For example, length is expressed in terms of $\sigma$ and energy in units of $\varepsilon$. In addition, temperature is expressed in units of $\varepsilon / k_{\mathrm{B}}$ and density in units of $\sigma^{-3}$. By definition, components of the local polarization are dimensionless quantities, as one can see from Eqs. (5.2)-(5.4).

Suppose now that we seek for a given $T$ that value of $\mu$ for which two phases " and " coexist. Henceforth, we adopt the convention that $\rho^{\prime}<\rho^{\prime \prime}$. If the densities $\rho^{\prime}$ and $\rho^{\prime \prime}$ of the two phases are sufficiently different, we expect that plots of $\Omega / V$ [calculated from Eq. (4.2)] versus $\mu$ (at fixed $T$ ) can be represented by lines of different (negative) slope; these lines intersect at the chemical potential at which the phases ' and " are at coexistence. Once convergence of the simultaneous solution of Eqs. (4.9) and (4.10) has been achieved, a new temperature $\widetilde{T}=T+\delta T$ and chemical potential $\widetilde{\mu}^{*}=\mu^{*}+\delta \mu^{*}$ are chosen, and the converged solutions of Eqs. (4.9) and (4.10) at $T$ and $\mu^{*}$ are taken as new starting solutions.

In practice, we take $\delta T=10^{-2}$ and $\delta \mu^{*}=10^{-1}$ for reduced temperatures $t=1-T / T_{\mathrm{c}} \lesssim 10^{-1}\left(T_{\mathrm{c}}\right.$ is the critical temperature); over the range $10^{-1} \lesssim t \lesssim 4 \times 10^{-2}$, the increments are reduced to about $\delta T=\delta \mu^{*} \simeq 10^{-2}$ to achieve convergence of the numerical solution of Eqs. (4.9) and (4.10); the increments are reduced further to $\delta T=\delta \mu^{*} \simeq 10^{-3}$ in the regime $4 \times 10^{-2}$ $\lesssim t \lesssim \times 10^{-2}$ as one approaches the critical point. Closer to the critical point, i.e., for $t \lesssim 10^{-2}$, it turns out that convergence of the iterative solution of Eqs. (4.9) and (4.10) can only be obtained if the increments $\delta T$ and $\delta \mu$ are reduced further by several orders of magnitude.

To save computer time as much as possible, we adopted a different approach in the near-critical regime $t \lesssim 10^{-2}$. First, we verified by a procedure described in detail in Ref. 36 that over the temperature range accessible through the above protocol, solutions of Eq. (4.9) can be described by

$$
\Delta \rho=\rho^{\prime \prime}-\rho^{\prime} \propto t^{\beta_{\mathrm{c}}},
$$

where $\beta_{\mathrm{c}}=\frac{1}{2}$ is the classical critical exponent governing the shape of the coexistence curve in the vicinity of the critical point. Hence, $\Delta \rho^{2}$ is a linear function of $T$ in the so-defined near-critical regime that can be fitted with $f(T)=a T+b$; thus, from the condition $f\left(T_{\mathrm{c}}\right)=0, T_{\mathrm{c}}=-b / a$ is readily obtained taking $a$ and $b$ as fit parameters.

\section{Isotropic square-well fluid confined by hard walls: A test case}

To validate our model and the numerical approach, we begin the presentation of our results by considering an isotropic bulk square-well fluid [i.e., $\varepsilon_{\mathrm{H}}=0$, see Eq. (2.4)]. Plots in Fig. 1 reveal that the coexistence curve obtained within the present DFT approach is in fairly good agreement with corresponding Gibbs-ensemble MC results. ${ }^{37}$ On the gas side of the coexistence curve, the agreement between both sets of data is excellent, whereas on the liquid side of the coexistence curve, there is a slightly larger disagreement between DFT and MC data. Nevertheless, the overall agreement is still

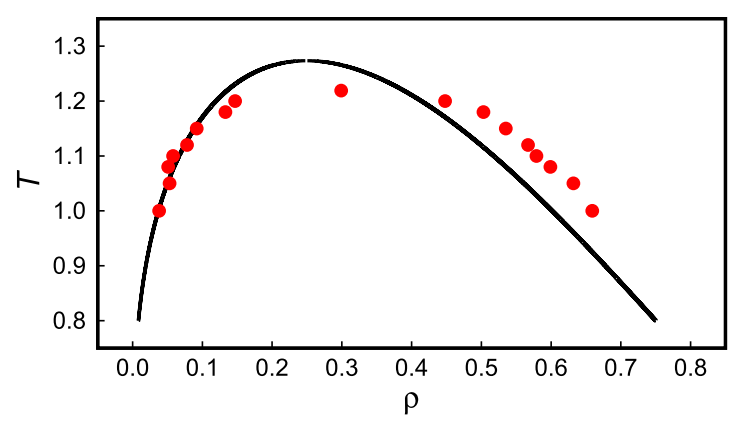

FIG. 1. Phase diagram of a bulk square-well fluid in $T-\rho$ representation; (black solid line) DFT data and (red filled circle) MC data from Ref. 37.

quite satisfactory. This holds in particular for the near-critical region where DFT overestimates $T_{\mathrm{c}}$ to some extent but underestimates the critical density $\rho_{\mathrm{c}}$ only slightly. This validates the mean-field treatment adopted here in combination with Eq. (2.3).

A more detailed comparison is possible employing data for the local density $\rho$ obtained by Henderson and van Swol earlier in molecular dynamics (MD) simulations. ${ }^{38}$ This comparison is made for $T=1.0$ and two bulk densities off coexistence in Figs. 2(a) and 2(b) (see Fig. 3). Both plots reflect layering of the fluid through damped oscillations of $\rho(z)$ where the period of the oscillations is about $\sigma$. At the lower density [see Fig. 2(a)], both DFT and MD data are in very good agreement with each other with a little bit of overshoot of the MD-generated density profile directly at the wall. At the higher density, the overshoot persists across the entire slit-pore [see Fig. 2(b)]. However, the period of the damped oscillations between both sets of data is in very good agreement and the disagreement between the amplitudes of the oscillations is still small.

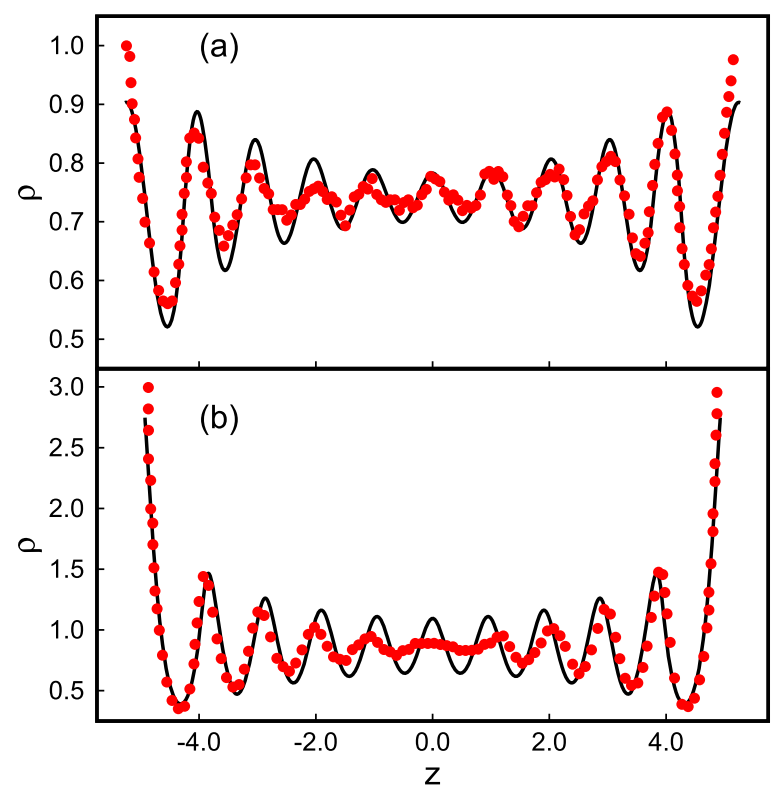

FIG. 2. Local density $\rho(z)$ as a function of position $z$ for a square-well fluid with isotropic attractions confined between hard walls $\left(\varepsilon^{\prime}=\varepsilon_{\mathrm{H}}=0\right)$ for a temperature $T=1.0$; (black solid line) DFT data and (red filled circle) MD data of Ref. 38; parts (a) and (b) of the figure correspond to bulk densities of $\rho_{\mathrm{b}}=0.741\left(s_{\mathrm{Z}}=10.549\right)$ and $\rho_{\mathrm{b}}=0.856\left(s_{\mathrm{Z}}=9.865\right)$, respectively. 


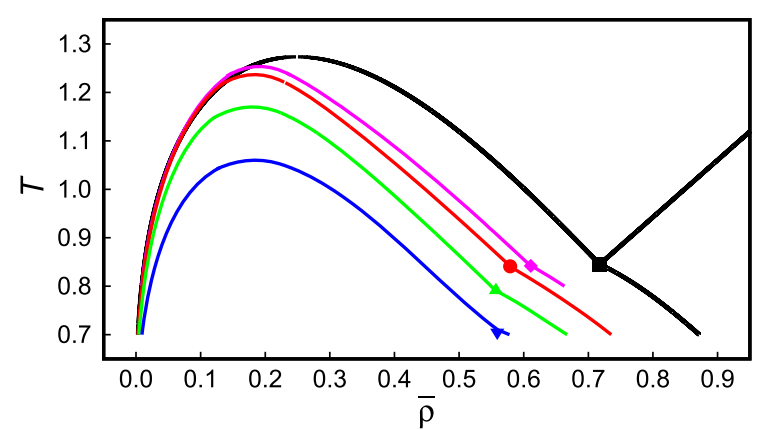

FIG. 3. Plots of phase diagrams for a square-well fluid with isotropic interactions confined between hard walls [i.e., $\varepsilon^{\prime}=0.00, \varepsilon_{\mathrm{H}}=0.25$; see Eqs. (2.4) and (2.8)]; critical end points $\left(T_{\text {cep }}, \rho_{\text {cep }}\right)$ are demarcated by symbols where (black square) bulk, i.e., $s_{\mathrm{Z}} \rightarrow \infty$; (magenta diamond) $s_{\mathrm{Z}}=15$; (red filled circle) $s_{\mathrm{Z}}=10$; (green triangle) $s_{\mathrm{Z}}=5$; and (blue triangle down) $s_{\mathrm{Z}}=3$. The critical line is only shown for the bulk fluid for the sake of clarity of the plot but would exist for all the curves shown.

In making the comparison between DFT and MD in Fig. 2, a couple of additional points need to be emphasised. The simulations carried out by Henderson and van $\mathrm{Swol}^{38}$ have been carried out for a fixed number density in the pore. To compare the MD results with the corresponding bulk phase diagram of the bulk square-well fluid, Henderson and van Swol estimate the bulk density by averaging their density profiles of the confined fluid around the pores' midpoint assuming that here packing effects are already small enough. More importantly, however, in the simulations of Henderson and van Swol, our Eq. (2.3) (motivated by the RPA approximation) is replaced by

$$
\varphi_{\mathrm{sw}}\left(r_{12}\right)=-\varepsilon \Theta\left(r_{12}-\sigma\right) \Theta\left(\lambda \sigma-r_{12}\right),
$$

cutting off contributions from attractions inside the hard core. Despite these differences, we conclude from plots presented in Fig. 2 that our DFT data are in very good agreement with the MD results obtained by Henderson and van Swol. ${ }^{38}$

Confining the square-well fluid with anisotropic attraction to a slit-pore with hard walls [i.e., $\varepsilon^{\prime}=0.00, \varepsilon_{\mathrm{H}}=0.25$; see Eqs. (2.4) and (2.8)] affects the phase behavior of the fluid in specific ways. First, one notices from plots in Fig. 3 that the critical temperature is depressed and the critical density is shifted to lower values (with respect to the bulk). These effects are known experimentally for a long time as demonstrated, for example, by Thommes and Findenegg. ${ }^{39}$ For $\mathrm{SF}_{6}$ confined to controlled-pore glasses (CPG) of nominal widths of $31 \mathrm{~nm}$ and $24 \mathrm{~nm}$, these authors measured a depression of the critical temperature in confinement of about $0.48 \mathrm{~K}$ and of $0.92 \mathrm{~K}$ relative to the bulk critical temperature of $\mathrm{SF}_{6}$, respectively; likewise, the critical density is shifted to lower values in confinement similar to what is found here in Fig. 3 (cf., Fig. 6 of Ref. 39). Similar critical-point shifts have been reported later by Morishige and Ito $^{40}$ for mesoporous silica materials of the MCM and SBA types where the pore width is much better defined than in the CPG materials employed earlier by Thommes and Findenegg. ${ }^{39}$

Moreover, as the confined square-well fluid is capable of forming a polar phase and because the walls themselves do not induce any order, a cep exists according to the discussion put forth in Sec. IV. One notices from plots in Fig. 3 that the

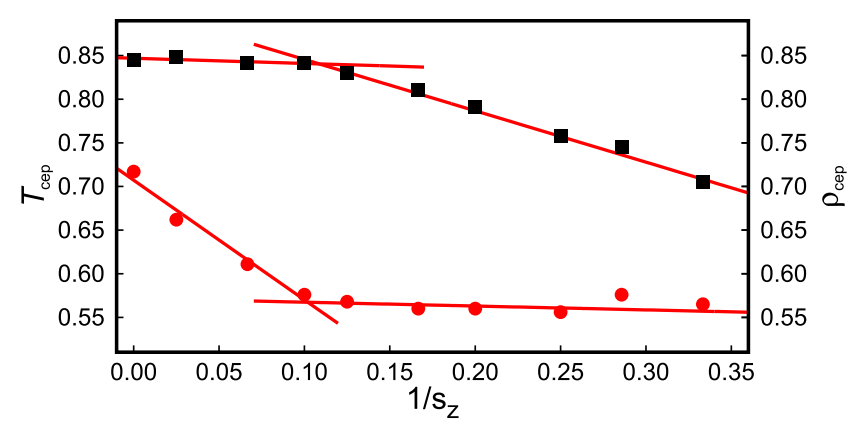

FIG. 4. Plots of temperature $T_{\text {cep }}$ (black square) and density $\rho_{\text {cep }}$ (red filled circle) of the critical end point as functions of the (inverse) pore width $1 / s_{\mathrm{Z}}$. Straight lines are fits to the discrete data points intended to guide the eye.

cep $\left(T_{\text {cep }}, \rho_{\text {cep }}\right)$ is shifted to lower temperatures and densities the more severely confined the fluid is. As also discussed in Sec. IV, at the cep, a line of critical points begins connecting isotropic and polar fluid states through a continuous (i.e., second-order) phase transition.

The dependence of $T_{\text {cep }}$ and $\rho_{\text {cep }}$ is illustrated by plots in Fig. 4. As one can also see from Fig. 4, there is a distinct difference between both plots. Although $T_{\text {cep }}$ becomes constant in the limit of sufficiently large pores (i.e., as $1 / s_{\mathrm{Z}} \rightarrow 0$ ), the opposite is true for $\rho_{\text {cep }}$ which tends to assume a plateau in the limit of narrow pores.

To promote a more detailed analysis based on the structure of the polar phase, we present in Fig. 5 plots of the local density $\rho$ where we vary the distance $s_{\mathrm{Z}}$ between the pore walls. In the narrowest pore $\left(s_{\mathrm{Z}}=5\right)$, we observe four peaks in the plot of $\rho$. Because of the symmetry of the fluid-wall interaction, the structure of $\rho$ is symmetric with respect to the pores' midplane located at $z=0$. This symmetry is preserved regardless of the pore width.

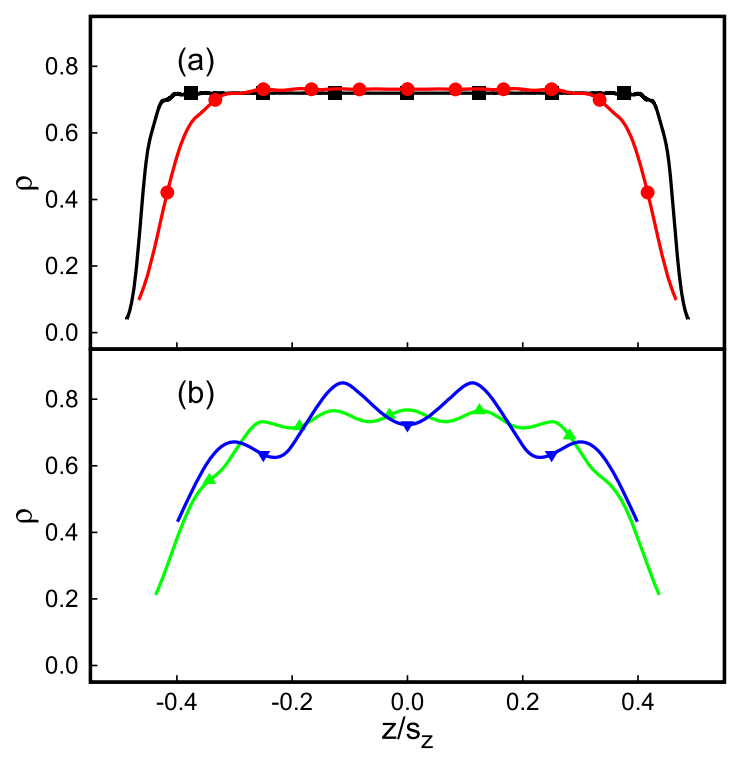

FIG. 5. The same as Fig. 2, but for a square-well fluid confined to slit-pores with hard walls; (a) (black square) $s_{\mathrm{Z}}=40$ and (red filled circle) $s_{\mathrm{Z}}=15$; (b) (green triangle) $s_{\mathrm{Z}}=8$ and (blue triangle down) $s_{\mathrm{Z}}=5$. Data are presented for the respective critical end points $\left(T_{\text {cep }}, \rho_{\text {cep }}\right)$ (see Fig. 4 ) that are listed in Table II. 
One also sees from Fig. 5 that for $s_{\mathrm{Z}} \gtrsim 10$ a bulk-like region centered on the pores' midpoint exists, which is characterized by an approximately constant part of the density profiles; as expected, this bulk-like region widens as $s_{\mathrm{Z}}$ increases because of the diminishing degree of confinement. Because the fluid cannot wet the walls on account of their hard nature, $\rho$ declines as $|z| \rightarrow s_{\mathrm{Z}} / 2$ [see Fig. 5] regardless of $s_{\mathrm{Z}}$.

The formation of a bulk-like region at the center of the pore for $s_{\mathrm{Z}} \gtrsim 15$ can be correlated with plots presented in Fig. 4. As $s_{\mathrm{Z}}$ increases, the portion of the confined fluid affected by the presence of the walls diminishes relative to the size of the bulk-like region of the pore fluid. This suggests that in this bulk-like region fluid molecules "experience" on average an attractive mean field that approaches an almost constant value if $s_{\mathrm{Z}}$ is sufficiently large.

This is also reflected by the observation from Fig. 4 that $T_{\mathrm{c}} \simeq 1.25$ for $s_{\mathrm{Z}} \gtrsim 15$ is not too far off $T_{\mathrm{c}} \simeq 1.27$ for the corresponding bulk fluid. Hence, increasing the pore width beyond $s_{\mathrm{Z}}=15$ does not shift the phase diagram appreciably along the $T$-axis with respect to the bulk. However, as the plots in Fig. 3 show, the coexistence curve of any confined fluid is narrower than that of the bulk phase. Consequently, for sufficiently large $s_{\mathrm{z}}$, the only way that the coexistence curve of the confined fluid can eventually approach that of the corresponding bulk system is by widening of the coexistence curve along the $\rho$-axis, and this is why we observe an increase of $\rho_{\text {cep }}$ with $s_{\mathrm{Z}}$ for sufficiently wide pores, whereas $T_{\text {cep }}$ is almost constant (see Fig. 4) for reasons already explained.

The opposite effect is observed in sufficiently narrow pores. Here, packing effects dominate the structure of the fluid (see Fig. 5). As one increases the pore width, the number of fluid layers increases, as plots in Fig. 5(b) show. However, the local density oscillates around an almost constant mean density as the entries for $\rho_{\text {cep }}$ in Table II and $s_{\mathrm{Z}}=5$ and 8 clearly show. However, the attractive mean field exerted by neighboring portions of the fluid differs markedly in narrow pores of different sizes. This causes $T_{\text {cep }}$ to depend sensitively on $s_{\mathrm{Z}}$ in narrow pores where the structure of the confined fluid is dominated by individual layers across the pore space.

\section{Impact of substrate anchoring}

We now turn to a detailed analysis of the phase behavior of a fluid confined between attractive walls where the walls themselves can orient the molecules in their vicinity. In other words, the formation of ordered polar phases is now not only driven by the thermodynamic state of the confined fluid but also directly by the presence of the walls. We focus

TABLE II. Temperature $T_{\text {cep }}$ and density $\rho_{\text {cep }}$ of the critical end point for various pore widths $s_{\mathrm{Z}}$.

\begin{tabular}{lcc}
\hline \hline$s_{\mathrm{Z}}$ & $T_{\text {cep }}$ & $\rho_{\text {cep }}$ \\
\hline 5 & 0.791 & 0.560 \\
8 & 0.830 & 0.568 \\
15 & 0.842 & 0.611 \\
40 & 0.848 & 0.662 \\
$\infty$ & 0.845 & 0.717 \\
\hline \hline
\end{tabular}

on the first three anchoring scenarios compiled in Table I and consider a pore of an intermediate width of $s_{\mathrm{Z}}=15$. In this system, a quasi-bulk-like region exists in which small vestiges of layering still persist across the pore; the interaction strength between fluid molecules and the walls is characterized by $\varepsilon^{\prime}=2.0$ to make sure that the molecules prefer to wet the walls to some extent. At the same time, we want to have a pore that is wide enough to illustrate that the polar phases do indeed possess long-range orientational order.

The phase behavior of the system is illustrated by the plots shown in Fig. 6. As one can see, the two-phase region is considerably wider than for a system with hard repulsive walls. The widening is mostly due to a shift of the coexistence line of liquid-like phases to higher densities. The critical densities have been shifted to significantly larger values compared with the hard-wall system, whereas the critical temperature appears to be almost unchanged. One also notices that the nature of the anchoring conditions is largely inconsequential except for relatively low $T$ where the inset in Fig. 6 reveals minute differences. In the vicinity of the critical point, one notices a weak shoulder on the gas side of the phase diagram. A similar feature has been observed for a Heisenberg fluid dissolved in an apolar solvent and exposed to a weak spatially homogeneous external magnetic field [see Fig. 9(a) of Ref. 41].

It is also instructive to consider the variation of the pore average polarization $\bar{P}=s_{\mathrm{z}}^{-1} \int \mathrm{d} z P(z)$ as a function of $T$ along the phase boundaries of the polar phase (cf., Fig. 6). Plots in Fig. 7 reveal that in the case of hard repulsive walls, $\bar{P}$ decays monotonically and drops to zero at the cep; for $T$ $\geq T_{\text {cep }}, \bar{P}=0$ until $T=T_{\text {c }}$. As we explain in Sec. IV, a cep cannot exist if the walls themselves anchor the molecules in specific ways. This notion is corroborated by the plots in Fig. 7 for all three anchoring scenarios considered. For the $o$ anchoring, we show plots of $\bar{P}$ for coexisting gas and polar liquid phases. One notices that both phases remain ordered all the way to the critical temperature where both sets of data merge as they must. Moreover, the insets in Figs. 6 and 7 are consistent with each other in which the specific anchoring has a small effect on the phase diagrams and $\bar{P}$ at low $T$,

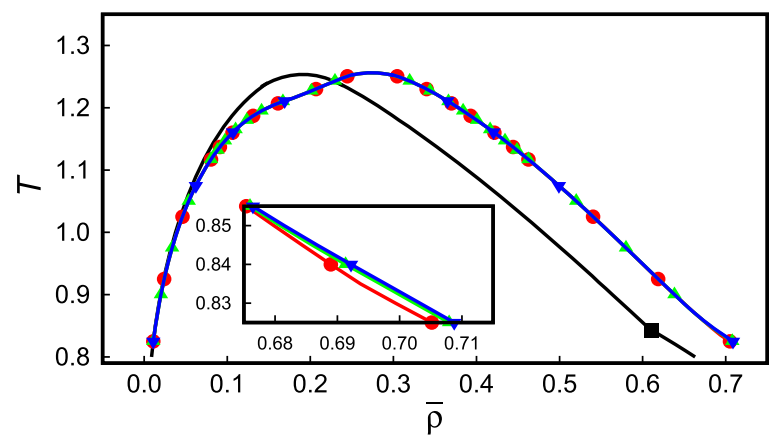

FIG. 6. The same as Fig. 3, but for a fluid confined between attractive walls separated by a distance $s_{\mathrm{Z}}=15$; (blue triangle down) parallel $(p)$ anchoring, (green triangle) orthogonal (o) anchoring, and (red filled circle) antiparallel $\left(a_{x}\right)$ anchoring. For comparison, the phase diagram for a slit-pore with hard repulsive walls is also shown (black square). The inset is an enhancement around the cep of the hard-wall system. 


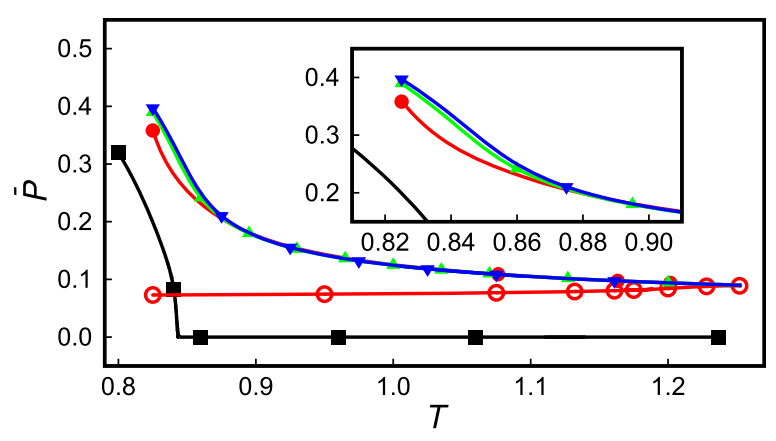

FIG. 7. Plots of the pore average polarization $\bar{P}$ as a function of temperature $T$ for a polar liquid-like phase confined between attractive walls $\left[\varepsilon^{\prime}=2.0\right.$; see Eq. (2.8)] with parallel ( $p$ ) (blue triangle down), orthogonal ( $o$ ) (green triangle), and antiparallel $\left(a_{x}\right)$ (red filled circle) anchoring (see Table I). For comparison, data for hard repulsive walls without specific anchoring are also shown (black square); in addition, results for the gas phase for the $a_{x}$ anchoring scenario (red open circle) are also presented. The inset is an enhancement of the plots in the main part of the figure.

whereas at higher $T$, the data are unaffected by the anchoring conditions.

The structure of the polar phase is illustrated by the plots in Fig. 8. If the walls of the slit-pore are hard, the local density follows a continuous curve that exhibits a more or less bulk-like midsection and decays as $|z| \rightarrow s_{\mathrm{z}} / 2$ similar to what we already saw in Fig. 5. If the wall is attractive, the contact value of $\rho$ in Fig. 8 is enhanced compared with the bulk-like midsection. It then decays until $\rho$ reaches a minimum at $|z| \simeq s_{\mathrm{z}} / 2-\sigma$ on account of packing effects. Beyond that minimum, $\rho$ increases again because now there is enough space between the wall and a reference particle; a reference molecule located in the range $s_{\mathrm{Z}} / 2-\lambda \sigma \leq|z| \leqslant s_{\mathrm{Z}} / 2-\sigma$ can more easily be surrounded by other molecules.

Directly at $|z|=s_{z} / 2-\lambda \sigma$, the attractive well of the fluid-wall potential vanishes discontinuously. Consequently, $\rho$ exhibits a similar discontinuity. Because the fluid-wall attraction vanishes, $\rho$ drops below the bulk value and continues to exhibit small oscillations around the bulk density $\rho_{\mathrm{b}} \simeq 0.74$. An interesting feature is illustrated by the insets in Fig. 8. As one can see from Fig. 8(a), sufficiently close to the walls, the local density is apparently insensitive to the anchoring at the

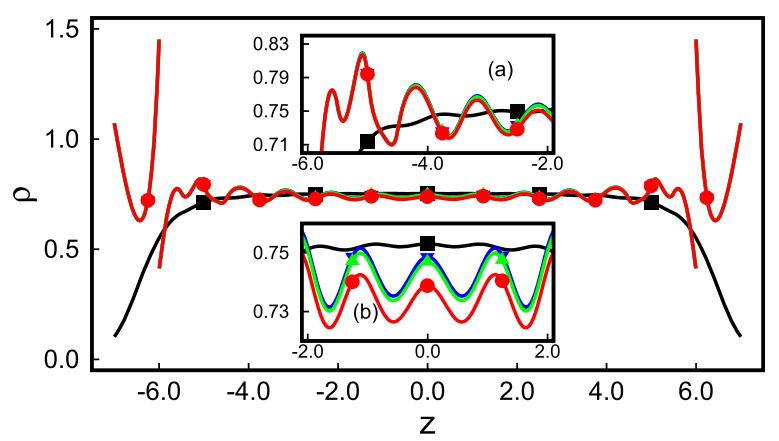

FIG. 8. The same as Fig. 2, but for attractive walls $\left[\varepsilon^{\prime}=2.0\right.$, see Eq. (2.8)] with parallel $(p)$ (blue triangle down), orthogonal (o) (green triangle), and antiparallel $\left(a_{x}\right)$ (red filled circle) anchoring (see Table I). For comparison, data for hard repulsive walls without specific anchoring are also shown (black square). Insets (a) and (b) are enhancements of various portions of the plots in the main part of the figure. Thermodynamic states are characterized by $T=0.83$ at the phase coexistence and $s_{\mathrm{Z}}=15$. walls. Here packing of the molecules must be controlled by their hard-core interaction. Around the pore's midplane (i.e., around $z=0$ ), minute differences in the amplitude of $\rho$ are discernible and the mean densities are slightly different depending on the anchoring. For example, the mean density is the highest and nearly the same for $p$ and $o$ anchoring, whereas it is the lowest for the $a_{x}$ anchoring. This indicates that $a_{x}$ anchoring destabilizes the confined fluid relative to the other two scenarios (see also Sec. VI).

Persisting oscillations across the entire pore space have been reported earlier by Greschek and Schoen who studied confined nematic phases of a liquid crystal by means of computer simulations. ${ }^{42}$ They have been attributed to packing effects when ordered structures have been formed. Similar observations have also been made by Jordanovic and Klapp $^{43,44}$ for confined dipolar fluids.

Plots of the local polarization $P$ in Fig. 9 corroborate these findings. Very close to the walls, $P$ turns out to be insensitive to the anchoring conditions. This is to be expected because the attraction of molecules by the closer wall is sufficiently strong, whereas the influence of the more remote one is negligible for $s_{\mathrm{Z}}=15$.

The orientation induced by the wall is then transmitted to portions of the fluid that do not directly interact with the nearest wall. This illustrates the long-range character of orientational order in our system. As one moves away from a wall, $P$ decays monotonically until one reaches the pore's midplane located at $z=0$. This is true regardless of the specific anchoring condition. However, the amount of order at the midplane is about 7\% higher for the $p$ as compared with the $o$ anchoring conditions (see inset in Fig. 9); the amount of order for the $a_{x}$ anchoring at the midplane is only $51 \%$ of that observed for the $p$ anchoring.

In the case of nonorienting hard walls, the polarization profiles qualitatively match the variation of the local density across the pore (cf., Figs. 8 and 9 ). That is, $P$ is the lowest in the immediate vicinity of the walls where $\rho$ is the lowest as well; the polarization then increases steadily toward the midplane where it attains its highest value. However, compared with $\rho$, which attains its plateau bulk value for $|z| \lesssim 4.0$ (see Fig. 8), the monotonic increase of $P$ is observed until $z=0$. This illustrates again the longrange character of orientational order as opposed to packing effects.

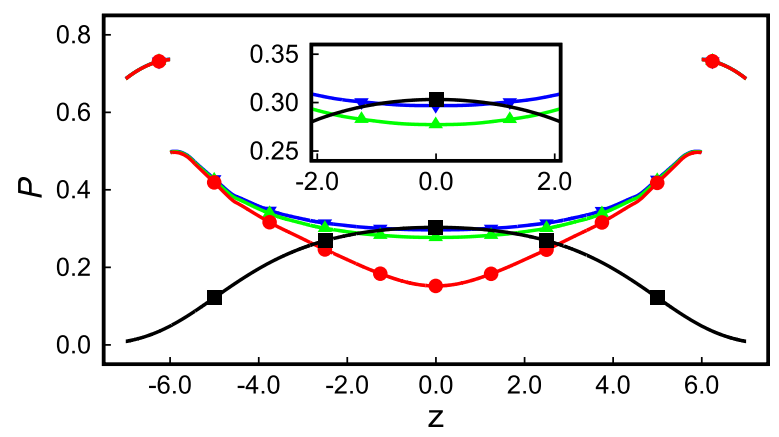

FIG. 9. Polarization profiles $P(z)$ as a function of position $z$ corresponding to the plots in Fig. 8. 
To gain deeper insight into the variation of orientational order across the pore, we present in Fig. 10 plots of Cartesian components of the local polarization vector $\boldsymbol{P}$ across the pore and for different anchoring scenarios compiled in Table I. As one might have anticipated, the plots in Fig. 10(a) indicate that in the case of $p$ anchoring the polarization is homogeneous in the sense that $P=P_{\mathrm{x}}$ [cf., Figs. 9 and 10(a)] and thus, $P_{\mathrm{y}}=P_{\mathrm{z}}$ $=0$.

This situation changes for the $o$ and $a_{x}$ anchoring scenarios as the respective plots in Figs. 10(b) and 10(c) reveal; in both cases, $P_{\mathrm{z}}=0$. For instance, as far as the $o$ anchoring condition is concerned, the plots in Fig. 10(b) show that

$$
P_{\mathrm{x}}(z)=P_{\mathrm{y}}(-z) \text {. }
$$

In other words, $\boldsymbol{P}$ lies in the x-y plane and rotates in this plane by an angle of $\frac{\pi}{2}$ as one moves from one wall to the other. As far as the $a_{x}$ anchoring scenario is concerned, the symmetry of $\boldsymbol{P}$ as described by Eq. (5.11) changes to

$$
\begin{aligned}
& P_{\mathrm{x}}(z)=-P_{\mathrm{x}}(-z), \\
& P_{\mathrm{y}}(z)=P_{\mathrm{y}}(-z) .
\end{aligned}
$$

To analyze the variation of the polarization for the more complex anchoring scenarios $o$ and $a_{x}$ [cf., Figs. 10(b) and 10(c)], we plot in Fig. 12 components of the local unit vector $\widehat{\boldsymbol{P}}^{\mathrm{T}}=\left(\widehat{P}_{\mathrm{x}}, \widehat{P}_{\mathrm{y}}, 0\right)$ as a function of position between the pore walls. The plots in Fig. 12 indicate that the reorientation for the $o$ and $a_{x}$ anchoring conditions follow in essence the same

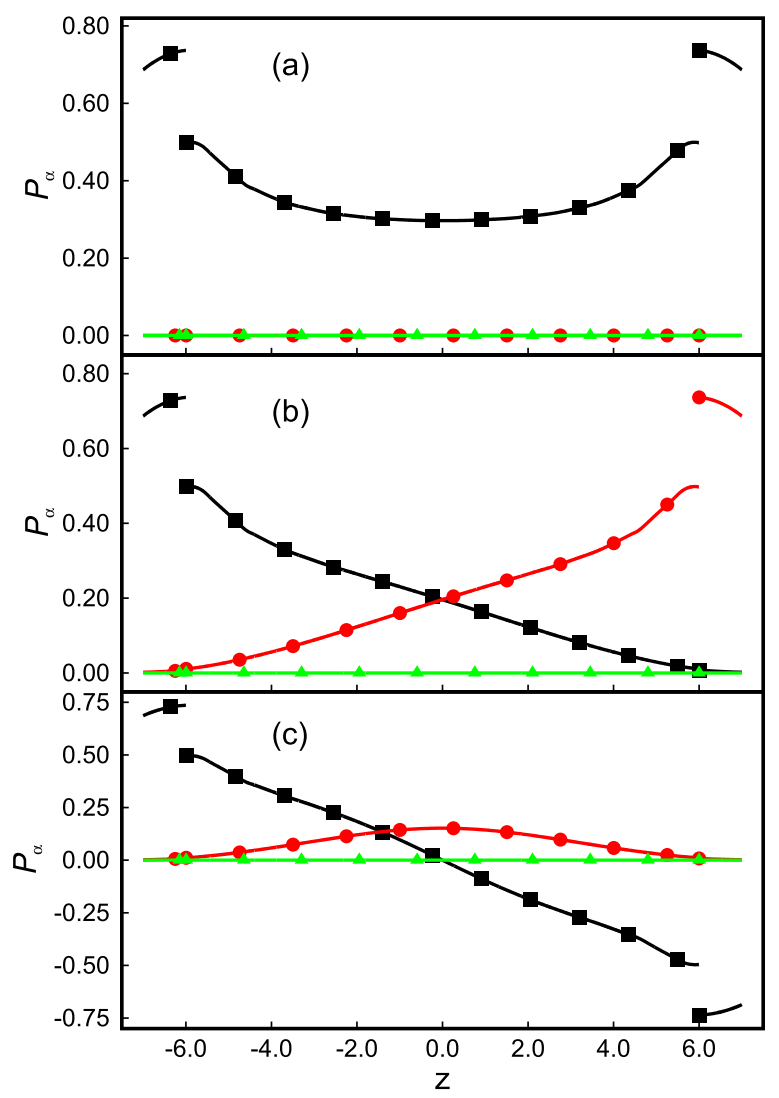

FIG. 10. The same as Fig. 9, but for Cartesian components of the local polarization vector $\boldsymbol{P}(z)$; (black square) $P_{\mathrm{x}}(z)$, (red filled circle) $P_{\mathrm{y}}(z)$, and (green triangle) $P_{\mathrm{z}}(z)$; (a) parallel $(p)$ anchoring, (b) orthogonal $(o)$ anchoring, and (c) antiparallel $\left(a_{x}\right)$ anchoring (see Table I).

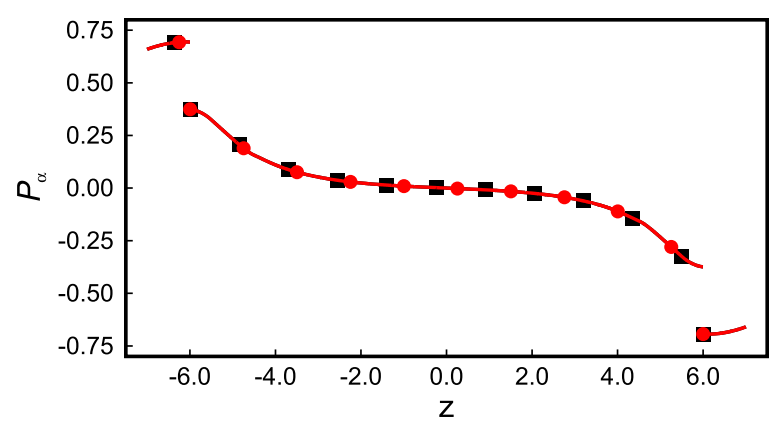

FIG. 11. Plots of components $P_{\alpha} \alpha=x$ (black square) ( $a_{x}$ anchoring) and $\alpha=z$ (red filled circle) ( $a_{z}$ anchoring) of the polarization $\boldsymbol{P}$ as a function of position $z$ between the walls of a slit pore $\left(s_{\mathrm{Z}}=15\right)$ at $T=0.90$ (cf., Figs. 6 and 7 and Table I).

general principle: starting with a perfect alignment of $\widehat{\boldsymbol{P}}$ with the positive $x$-axis at $z=-s_{z} / 2, \widehat{\boldsymbol{P}}$ passes through a state in which it is perfectly aligned with the positive $y$-axis. The difference is that this perfect alignment with the positive $y$-axis is attained at $z=s_{\mathrm{Z}} / 2$ for the $o$ anchoring condition, whereas it is assumed at the pore's midplane (i.e., at $z=0$ ) as far as the $a_{x}$ anchoring scenario is concerned. Thus, the twist of $\widehat{\boldsymbol{P}}$ is twice as large that in the $a_{x}$ as opposed to the $o$ anchoring. As rotating $\widehat{\boldsymbol{P}}$ costs free energy, we surmise that the larger twist in the $a_{x}$ anchoring case explains the overall reduction of $P$ in Fig. 9 compared with both the $p$ and $o$ anchoring conditions. Note, that despite the discontinuities in density (see Fig. 8) and polarization profiles (see Fig. 10) the unit vector $\widehat{\boldsymbol{P}}$ changes continuously along the $z$-axis as it should. However, according to the arguments presented in Sec. V A, we
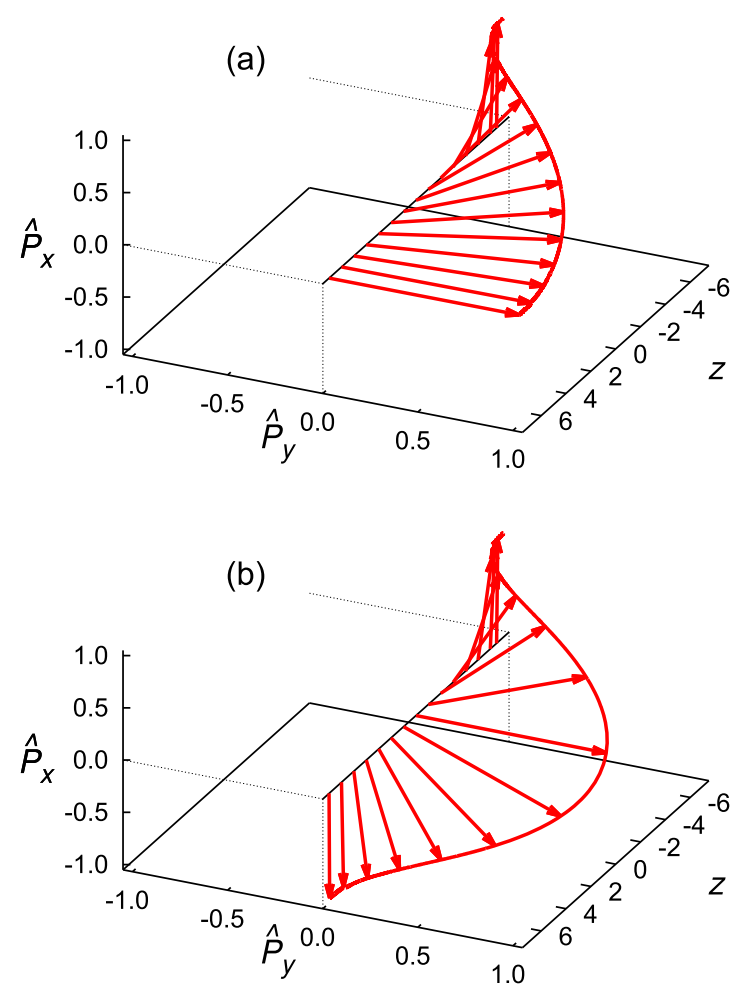

FIG. 12. Plots of components of the unit vector $\widehat{\boldsymbol{P}}(z)$ as a function of the position $z$ between the pore walls. Red arrows indicate the direction of $\widehat{\boldsymbol{P}}(z)$ in the $x-y$ plane; (a) orthogonal $(o)$ anchoring [see Fig. 10(b)] and (b) antiparallel $\left(a_{x}\right)$ anchoring [see Fig. 10(c)]. 
would expect the polarization profiles to be identical in shape for all antiparallel anchoring scenarios (i.e., for $a_{x}$ and $a_{z}$, see Table I). That this is indeed the case and is illustrated by plots of $P_{\mathrm{x}}$ and $P_{\mathrm{z}}$ presented in Fig. 11.

The continuous variation of $\widehat{\boldsymbol{P}}$ can be rationalized as follows. As one can see from the plots in Fig. 9, the discontinuous change in $P_{\alpha}$ ( $\alpha=\mathrm{x}$ or $\mathrm{y}$ ) near a wall occurs only in one of the two components, whereas the other component of the polarization vector vanishes. This implies that the magnitude of the discontinuities seen in Fig. 10 is the same as the one in $P$. On account of the definition of $\widehat{\boldsymbol{P}}$, this latter quantity should be free of any discontinuity which is, in fact, what the plots in Fig. 12 reveal.

\section{DISCUSSION AND CONCLUSIONS}

In this work we reconsider a three-dimensional Heisenberg fluid confined to a slit-pore. We employ classical DFT to investigate the phase behavior of the confined fluid as well as its orientational and positional structure unlike in Paper $\mathrm{I}^{14}$ of this study where the latter had been neglected altogether. To account for positional order in the confined fluid, we adopt FMT that allows one to describe the formation of layers in confined fluids ${ }^{45}$ or fluids next to a wall. ${ }^{24}$ We consider two types of pore walls: non-orienting (hard) walls and walls that can themselves orient molecules in their vicinity on account of energetic features of the external field representing the pore walls.

The model employed here differs from that considered in Paper I of this series of manuscripts ${ }^{14}$ in that interactions between the isotropic cores of a pair of molecules are of short range. Specifically, we utilize a square-well potential where the width of the attractive well is given by $\lambda=\frac{3}{2}$. Thus, intermolecular interactions are cut off if the distance between a pair of molecules exceeds 1.5 times the diameter of their hard repulsive cores.

To model the anisotropic intermolecular interactions, we adopt an orientation-dependent square-well potential describing the interaction between a pair of classical spins in the classical three-dimensional Heisenberg model. Depending on the thermodynamic conditions, this model fluid is capable of forming an ordered polar phase in addition to the more conventional gas and isotropic liquid phases in the bulk. ${ }^{5-10,35}$

There is a threefold reason to adopt this simple yet sufficiently complex model. First, on account of the short-range nature of the attractive interactions, the phase boundaries of (isotropic or polar) liquid phases are shifted to lower densities that would make this model easily amenable to computer simulation approaches involving, for example, grand canonical Monte Carlo techniques. Second, the simple form of the square-well potential enables one to integrate out some degrees of freedom analytically, as is demonstrated, for example, in Appendix A of this work.

Third and perhaps most importantly, the short-range character of the anisotropic interactions permits one to study the formation of ordered polar phases without requiring more sophisticated techniques. For example, it has been demonstrated some time ago ${ }^{1}$ that the free-energy density of a dipolar fluid in an ordered phase depends on the shape of the sample considered and on the dielectric permittivity. The present model avoids these additional complications. Nonetheless, it should be noted that the general topologies of phase diagrams of dipolar fluids and of the Heisenberg fluid are the same (c.f., Fig. 15 of Ref. 1 and Fig. 1 of Ref. 10).

Here we focus on an intermediate coupling strength of the spin-spin interaction. The topology of the corresponding phase diagram turns out to be of type I in the terminology introduced by Cattes et al., ${ }^{41}$ that is, a gas coexists with a polar liquid at low $T$, whereas a gas phase and an isotropic liquid coexist at higher $T$; polar and isotropic liquids are separated by a line of critical points ending at a cep at which the critical line joins the phase boundaries of the high-density (isotropic and polar) liquid phases.

If under these conditions the Heisenberg fluid is confined to a slit-pore with hard, non-orienting walls, the general topology of the phase diagram prevails. However, details differ depending on the degree of confinement (i.e., the pore width). In general, the critical point, at which gas and isotropic liquid become indistinguishable, appears to be shifted to lower temperatures. The depression of the critical temperature is accompanied by a shift of the critical density to lower values (see Fig. 3). This shift is related to packing phenomena that could not be accounted for in Paper I of this study, ${ }^{14}$ in which we ignored the inhomogeneity of the confined fluid altogether. A shift of the critical point as observed here is in excellent qualitative agreement with experimental findings. ${ }^{39,40}$

A new result observed in the present work is a shift of the cep that chaperons the pore-size related shift of the critical point. Here two regimes of pore widths can be discerned. In the limit of large pores, $T_{\text {cep }}$ becomes approximately constant, whereas $\rho_{\text {cep }}$ increases steadily with increasing pore width.

We attribute this to the fact that in sufficiently wide pores a bulk-like region of homogeneous density exists. In this bulklike region, the attractive mean-field "experienced" by a fluid molecule on account of its neighbors is approximately the same. Thus the critical temperature of a fluid in a wide pore is almost the same as in the bulk. Thus, as packing effects play only a subdominant role in wide pores, the phase boundary of the confined liquid phases shifts back to its location in the bulk such that $\rho_{\text {cep }}$ increases.

If the pores are small, the opposite is found: $T_{\text {cep }}$ decreases monotonically with decreasing pore width, whereas $\rho_{\text {cep }}$ becomes nearly pore-size independent. In this regime, the confined fluid is highly inhomogeneous as reflected by individual layers; they correspond to peaks in the local density. Apparently, the packing within each layer does not depend on the pore width and only the number of these layers changes with the pore width. That packing features do not depend much on $s_{\mathrm{Z}}$ can probably be linked to the fact that at coexistence the (hard) walls cannot be wet by the fluid. However, with varying $s_{\mathrm{Z}}$, the mean-field caused by the attractive intermolecular interactions varies substantially. We ascribe the change of $T_{\text {cep }}$ with $s_{\mathrm{Z}}$ to this varying mean field.

If the external field itself is capable of orienting the molecules in specific ways, the cep is suppressed and isotropic phases cannot form. We demonstrate here that this is because 
of the specific anchoring conditions. As we argue, the consistency relation given in Eq. (4.10) cannot be satisfied by nonzero expansion coefficients $\left\{\alpha_{1 m}\right\}$ if the external potential $\Phi_{\text {ext }}$ has an explicitly orientation-dependent component. Because a cep cannot exist, a polar liquid phase is stable to a critical point of gas-polar liquid coexistence. At this critical point, it is no longer possible to discern a gas phase from a polar liquid phase implying that even the gas exhibits an albeit small degree of order at lower temperatures which is indeed what we find. Our findings also explain a similar suppression of a cep in cases in which the Heisenberg fluid is exposed to a spatially homogeneous external magnetic field considered in Ref. 41.

Last but not least, explicit anchoring scenarios can be either homogeneous or inhomogeneous. In the homogeneous case where the energetic discrimination of molecules is the same at both walls, the degree of order near each wall just decays as one approaches the midplane of the pore. This is due to the interplay between thermal energy of the fluid and the short-range character of the fluid-wall interaction; that this order does not completely vanish beyond the range of fluid-wall interactions illustrates its long-range character in the confined fluid.

If inhomogeneous anchoring is concerned, the energetic discrimination of the molecular orientation at one wall differs from that at the other. Thus, in an ordered phase, the molecules have to reorient themselves as one moves from one wall to the other. The reorientation process takes place in the $x-y$ plane for the two inhomogeneous anchoring scenarios considered $(o$ and $\left.a_{x}\right)$. However, for the anchoring conditions, the twist of the unit vector $\widehat{\boldsymbol{P}}$ associated with the polarization is twice of that observed for the $o$ anchoring scenario. Therefore, orientational order is usually weaker for the $a_{x}$ as opposed to the $o$ anchoring conditions.

\section{ACKNOWLEDGMENTS}

Two of us (S.M.W. and M.S.) acknowledge support from the Deutsche Forschungsgemeinschaft through the International Graduate Research Training Group 1524 "Selfassembled nanostructures at interfaces."

\section{APPENDIX A: THE SQUARE-WELL INTERACTION POTENTIAL IN CYLINDRICAL COORDINATES}

As we explain in Sec. III B, the specific functional form of $\Phi_{\text {ext }}$ suggests that the generic singlet distribution function can be decomposed according to Eq. (3.7). This implies that the free-energy contribution of the square well attraction in the reference system can be cast as

$$
\mathcal{F}_{\mathrm{sW}}=\frac{1}{2} \int \mathrm{d} \boldsymbol{r}_{1} \mathrm{~d} \boldsymbol{r}_{2} \rho\left(z_{1}\right) \rho\left(z_{2}\right) \varphi_{\mathrm{sw}}\left(r_{12}\right) .
$$

Because of the cylindrical symmetry of the fluid confined to a slit-pore, we introduce cylindrical coordinates which permit us to rewrite Eq. (A1) as

$$
\begin{aligned}
\mathcal{F}_{\mathrm{sw}}= & \frac{1}{2} \int \mathrm{d} z_{1} \int \mathrm{d} a_{1} a_{1} \mathrm{~d} \gamma_{1} \int \mathrm{d} z_{2} \int \mathrm{d} a_{12} a_{12} \mathrm{~d} \gamma_{12} \\
& \times \rho\left(z_{1}\right) \rho\left(z_{2}\right) \varphi_{\mathrm{sw}}\left(r_{12}\right),
\end{aligned}
$$

where $\left(a_{1}, \gamma_{1}\right)^{\mathrm{T}}$ is the projection of $\boldsymbol{r}_{1}$ onto the $x-y$ plane and a similar meaning has been adopted for $a_{12}$ and $\gamma_{12}$ with respect to the distance vector $\boldsymbol{r}_{12}$. In these coordinates, the integration over $a_{1} \mathrm{~d} a_{1} \mathrm{~d} \gamma_{1}$ can trivially be carried out and gives the area $A$ of a solid substrate; likewise, integrating over $\mathrm{d} \gamma_{12}$ gives a prefactor of $2 \pi$.

Because of the transformation to cylindrical coordinates, we need to replace $\varphi_{\text {sw }}$ in Eq. (2.3) by

$$
\begin{aligned}
\varphi_{\mathrm{sW}}\left(a_{12},\left|z_{1}-z_{2}\right|\right)= & -\varepsilon \Theta\left(\lambda \sigma-\sqrt{a_{12}^{2}+\left|z_{1}-z_{2}\right|^{2}}\right) \\
& \times \Theta\left(\lambda \sigma-\left|z_{1}-z_{2}\right|\right) .
\end{aligned}
$$

Therefore,

$$
\begin{aligned}
& \int \mathrm{d} a_{12} a_{12} \varphi_{\mathrm{sw}}\left(a_{12},\left|z_{1}-z_{2}\right|\right) \\
& =-\varepsilon \Theta\left(\lambda \sigma-\left|z_{1}-z_{2}\right|\right) \int_{\left|z_{1}-z_{2}\right|}^{\lambda \sigma} \mathrm{d} u u \\
& =-\frac{\varepsilon}{2}\left[(\lambda \sigma)^{2}-\left|z_{1}-z_{2}\right|^{2}\right] \Theta\left(\lambda \sigma-\left|z_{1}-z_{2}\right|\right),
\end{aligned}
$$

where we transformed variables according to $u=$ $\sqrt{a_{12}^{2}+\left|z_{1}-z_{2}\right|^{2}}$. Therefore,

$$
\begin{aligned}
\tilde{\varphi}_{\mathrm{sw}}\left(\left|z_{1}-z_{2}\right|\right)= & -\frac{\pi \varepsilon}{2}\left[(\lambda \sigma)^{2}-\left|z_{1}-z_{2}\right|^{2}\right] \\
& \times \Theta\left(\lambda \sigma-\left|z_{1}-z_{2}\right|\right) .
\end{aligned}
$$

Thus, Eqs. (A2) and (A5) eventually allow us to express $\mathcal{F}_{\text {sw }}$ in its final form given in Eq. (3.11).

\section{APPENDIX B: EFFICIENT EVALUATION OF INTEGRALS INVOLVING THE ORIENTATION-DISTRIBUTION FUNCTION}

An inspection of Eq. (4.10) reveals that we need to solve integrals of the form

$$
\begin{aligned}
\mathcal{J}_{0}(z) & =\int \mathrm{d} \omega \Psi(z, \omega), \\
\mathcal{J}_{1}(z) & =\int \mathrm{d} \omega \mathcal{Y}_{10}(\omega) \Psi(z, \omega) \\
& =\sqrt{\frac{3}{4 \pi}} \int \mathrm{d} \omega \cos \theta \Psi(z, \omega), \\
\mathcal{J}_{2}(z) & =\int \mathrm{d} \omega \operatorname{Re} \mathcal{Y}_{11}(\omega) \Psi(z, \omega) \\
& =-\sqrt{\frac{3}{8 \pi}} \int \mathrm{d} \omega \sin \theta \cos \phi \Psi(z, \omega), \\
\mathcal{J}_{3}(z) & =\int \mathrm{d} \omega \operatorname{Im} \mathcal{Y}_{11}(\omega) \Psi(z, \omega) \\
& =-\sqrt{\frac{3}{8 \pi}} \int \mathrm{d} \omega \sin \theta \sin \phi \Psi(z, \omega),
\end{aligned}
$$

which can be solved efficiently using Bessel functions as we shall demonstrate below. 
We begin with Eq. (B1a) which can be rewritten as

$$
\begin{aligned}
\mathcal{J}_{0}(z)= & \int_{0}^{\pi} \mathrm{d} \theta \sin \theta \exp [A(z) \cos \theta] \\
& \times \int_{0}^{2 \pi} \mathrm{d} \phi \exp [R(z) \sin \theta \cos \phi+I(z) \sin \theta \sin \phi],
\end{aligned}
$$

where

$$
\begin{aligned}
A(z) & \equiv-\varepsilon^{\prime} \sum_{k=1}^{2} \varphi_{\mathrm{sw}}\left(\Delta z^{(k)}\right) \cos \alpha^{(k)} \\
& +\sqrt{\frac{16 \pi}{3}} \int \mathrm{d} z^{\prime} \rho\left(z^{\prime}\right) \alpha_{10}\left(z^{\prime}\right) \widetilde{\varphi}_{\mathrm{sw}}\left(\left|z-z^{\prime}\right|\right), \\
R(z) \equiv & -\varepsilon^{\prime} \sum_{k=1}^{2} \varphi_{\mathrm{sw}}\left(\Delta z^{(k)}\right) \sin \alpha^{(k)} \cos \beta^{(k)} \\
& -\sqrt{\frac{32 \pi}{3}} \int \mathrm{d} z^{\prime} \rho\left(z^{\prime}\right) \operatorname{Re} \alpha_{11}\left(z^{\prime}\right) \widetilde{\varphi}_{\mathrm{sw}}\left(\left|z-z^{\prime}\right|\right), \\
I(z) \equiv & -\varepsilon^{\prime} \sum_{k=1}^{2} \varphi_{\mathrm{sw}}\left(\Delta z^{(k)}\right) \sin \alpha^{(k)} \sin \beta^{(k)} \\
& +\sqrt{\frac{32 \pi}{3}} \int \mathrm{d} z^{\prime} \rho\left(z^{\prime}\right) \operatorname{Im} \alpha_{11}\left(z^{\prime}\right) \widetilde{\varphi}_{\mathrm{sw}}\left(\left|z-z^{\prime}\right|\right),
\end{aligned}
$$

and Eqs. (2.8)-(2.11) and (4.7) (see also entries in Table I) have also been used. Henceforth, we shall drop the argument of $A, R$, and $I$ to simplify the notation.

We can rewrite Eq. (B2) in a slightly different form using the identity

$$
a \cos x+b \sin x=\sqrt{a^{2}+b^{2}} \cos (x-\gamma),
$$

where $\cos \gamma \equiv a / \sqrt{a^{2}+b^{2}}, \sin \gamma=b / \sqrt{a^{2}+b^{2}}$; in the present case, $a=R \sin \theta, b=I \sin \theta$, and $x=\phi$. The identity can easily be verified using the addition theorem for trigonometric functions. Using in addition, that $\sin \theta \geq 0$ in the interval $[0, \pi]$ and that

$$
\int_{0}^{2 \pi} \mathrm{d} \xi f[\cos (\xi+\nu)]=2 \int_{0}^{\pi} \mathrm{d} \xi f(\cos \xi)
$$

where $f$ is an arbitrary function and $v$ is a phase shift, we can rewrite Eq. (B2) as

$\mathcal{J}_{0}(z)=2 \int_{0}^{\pi} \mathrm{d} \theta \sin \theta \mathrm{e}^{A \cos \theta} \int_{0}^{\pi} \mathrm{d} \phi \exp \left[\sqrt{R^{2}+I^{2}} \sin \theta \cos \phi\right]$.

The second integral in the previous expression is a modified Bessel function of the first kind. ${ }^{26}$ It has an integral representation [see Eq. (9.6.19) of Ref. 26],

$$
\mathcal{I}_{n}(\zeta)=\frac{1}{\pi} \int_{0}^{\pi} \mathrm{d} \xi \exp (\zeta \cos \xi) \cos (n \xi),
$$

where $\zeta=x+i y$ is a complex number in general $(x, y \in \mathbb{R})$. With this definition, we can rewrite Eq. (B6) as

$$
\mathcal{J}_{0}(z)=2 \pi \int_{0}^{\pi} \mathrm{d} \theta \sin \theta \mathrm{e}^{A \cos \theta} \mathcal{I}_{0}\left(\sqrt{R^{2}+I^{2}} \sin \theta\right) .
$$

The charm of this representation of the integral $\mathcal{J}_{0}$ lies in the fact that besides the integral representation in Eq. (B7) there is also an equivalent series representation of the modified Bessel function of the first kind. It can be cast as [see Eq. (6.9.10) of Ref. 26]

$$
\mathcal{I}_{n}(\zeta)=\sum_{m=0}^{\infty} \frac{1}{m !(n+m) !}\left(\frac{\zeta}{2}\right)^{2 m+n} .
$$

With the aid of Eq. (B9), we can rewrite the previous expression for $\mathcal{J}_{0}$ as

$$
\mathcal{J}_{0}(z)=2 \pi \sum_{m=0}^{\infty} \frac{1}{(m !)^{2}}\left(\frac{\sqrt{R^{2}+I^{2}}}{2}\right)^{2 m} \int_{-1}^{1} \mathrm{~d} x\left(1-x^{2}\right)^{m} \mathrm{e}^{A x},
$$

where $x \equiv \cos \theta$. Using the binomial theorem, it is easy to verify that

$$
\left(1-x^{2}\right)^{m}=\sum_{k=0}^{m}\left(\begin{array}{c}
m \\
k
\end{array}\right)(-1)^{k} x^{2 k}
$$

and therefore,

$\mathcal{J}_{0}(z)=2 \pi \sum_{m=0}^{\infty} \frac{1}{(m !)^{2}}\left(\frac{\sqrt{R^{2}+I^{2}}}{2}\right)^{2 m} \sum_{k=0}^{m}\left(\begin{array}{l}m \\ k\end{array}\right)(-1)^{k} \int_{-1}^{1} \mathrm{~d} x x^{2 k} \mathrm{e}^{A x}$

in final form.

Turning now to a discussion of the remaining three integrals $\mathcal{J}_{1}-\mathcal{J}_{3}$ [see Eqs. (B1b)-(B1d)], one realizes that by similar mathematical manipulations these can be cast as

$$
\mathcal{J}_{1}(z)=\sqrt{3 \pi} \int_{0}^{\pi} \mathrm{d} \theta \sin \theta \cos \theta \mathrm{e}^{A \cos \theta} \mathcal{I}_{0}\left(\sqrt{R^{2}+I^{2}} \sin \theta\right),
$$

$$
\frac{\mathcal{J}_{2}(z)}{\cos \gamma}=-\sqrt{\frac{3 \pi}{2}} \int_{0}^{\pi} \mathrm{d} \theta \sin ^{2} \theta \mathrm{e}^{A \cos \theta} \mathcal{I}_{1}\left(\sqrt{R^{2}+I^{2}} \sin \theta\right),
$$

$$
\mathcal{J}_{3}(z)=\mathcal{J}_{2}(z) \tan \gamma
$$

Using exactly the same reasoning that eventually led to Eq. (B12), it is possible to verify that the analogous expressions for $\mathcal{J}_{1}$ and $\mathcal{J}_{2}$ are given by

$$
\begin{aligned}
\mathcal{J}_{1}(z)= & \sqrt{3 \pi} \sum_{m=0}^{\infty} \frac{1}{(m !)^{2}}\left(\frac{\sqrt{R^{2}+I^{2}}}{2}\right)^{2 m} \\
& \times \sum_{k=0}^{m}\left(\begin{array}{c}
m \\
k
\end{array}\right)(-1)^{k} \int_{-1}^{1} \mathrm{~d} x x^{2 k+1} \mathrm{e}^{A x},
\end{aligned}
$$




$$
\begin{aligned}
\frac{\mathcal{J}_{2}(z)}{\cos \gamma}= & -\sqrt{\frac{3 \pi}{2}} \sum_{m=0}^{\infty} \frac{1}{m !(m+1) !}\left(\frac{\sqrt{R^{2}+I^{2}}}{2}\right)^{2 m+1} \\
& \times \sum_{k=0}^{m+1}\left(\begin{array}{c}
m+1 \\
k
\end{array}\right)(-1)^{k} \int_{-1}^{1} \mathrm{~d} x x^{2 k} \mathrm{e}^{A x} .
\end{aligned}
$$

At this stage, a distinction of cases is required. In general, we need to consider four different situations.

\section{Isotropic phase}

We begin with the simplest case in which $A=R=I=0$. In this case, only $m=k=0$ needs to be considered in Eqs. (B12)(B14b). It is therefore clear that $\mathcal{J}_{0}=4 \pi$. From Eq. (B14a), it follows that $\mathcal{J}_{1}=0$ because the integrand is an odd function of $x$ and the range of integration is symmetric with respect to $x=0$. Similarly, $\mathcal{J}_{2}=0$ because for $m=0$, and $R=I=0$, the prefactor $\sqrt{R^{2}+I^{2}}=0$. Because of Eq. (B13c), $\mathcal{J}_{3}=0$.

\section{Polarization in the $x-y$ plane}

Next, consider $A=0$ but $R$ and/or $I$ are nonzero. In this case, it follows from Eq. (B12) that

$\mathcal{J}_{0}(z)=4 \pi \sum_{m=0}^{\infty} \frac{1}{(m !)^{2}}\left(\frac{\sqrt{R^{2}+I^{2}}}{2}\right)^{2 m} \sum_{k=0}^{m}\left(\begin{array}{l}m \\ k\end{array}\right) \frac{(-1)^{k}}{2 k+1}$.

From Eq. (B14a), one realizes that $\mathcal{J}_{1}=0$ for the same reason given for case $i$. For the remaining integral, it follows from Eq. (B14b) that

$$
\begin{aligned}
\frac{\mathcal{J}_{2}(z)}{\cos \gamma}= & -\sqrt{6 \pi} \sum_{m=0}^{\infty} \frac{1}{m !(m+1) !}\left(\frac{\sqrt{R^{2}+I^{2}}}{2}\right)^{2 m+1} \\
& \times \sum_{k=0}^{m+1}\left(\begin{array}{c}
m+1 \\
k
\end{array}\right) \frac{(-1)^{k}}{2 k+1} .
\end{aligned}
$$

From Eq. (B13c), it then follows that $\mathcal{J}_{3} \neq 0$.

\section{Polarization in z-direction}

If $A \neq 0$ but $R=I=0$, it is easy to verify from Eq. (B12) that

$$
\mathcal{J}_{0}(z)=4 \pi \frac{\sinh A}{A}
$$

because only the term for $m=0$ survives. The same is true in Eq. (B14a) and one finds

$$
\mathcal{J}_{1}(z)=\sqrt{12 \pi}\left(\frac{\cosh A}{A}-\frac{\sinh A}{A^{2}}\right) .
$$

Because for $m \geq 0,\left(R^{2}+I^{2}\right)^{(2 m+1) / 2}=0, \mathcal{J}_{2}=\mathcal{J}_{3}=0$.

\section{Polarization in arbitrary direction}

The most complicated case to be considered is the one characterized by $A \neq 0$ and nonvanishing $R$ and/or $I$. An inspection of Eqs. (B12), (B14a), and (B14b) reveals after some straightforward albeit tedious algebraic manipulations that

$$
\begin{aligned}
\mathcal{J}_{0}(z)= & 4 \pi \sum_{m=0}^{\infty} \frac{1}{(m !)^{2}}\left(\frac{\sqrt{R^{2}+I^{2}}}{2}\right)^{2 m} \sum_{k=0}^{m}\left(\begin{array}{l}
m \\
k
\end{array}\right)(-1)^{k} \\
& \times \sum_{i=0}^{2 k}(-1)^{i}\left(\frac{1}{A}\right)^{2 k-i+1} \frac{(2 k) !}{i !} \begin{cases}\sinh A, \quad i \text { even, } \\
\cosh A, \quad i \text { odd, }\end{cases} \\
\mathcal{J}_{1}(z)= & \sqrt{12 \pi} \sum_{m=0}^{\infty} \frac{1}{(m !)^{2}}\left(\frac{\sqrt{R^{2}+I^{2}}}{2}\right)^{2 m} \sum_{k=0}^{m}\left(\begin{array}{l}
m \\
k
\end{array}\right)(-1)^{k} \\
& \times \sum_{i=0}^{2 k+1}(-1)^{i+1}\left(\frac{1}{A}\right)^{2 k-i+2} \frac{(2 k+1) !}{i !} \begin{cases}\sinh A, & i \text { even }, \\
\cosh A, & i \text { odd },\end{cases}
\end{aligned}
$$

$$
\begin{aligned}
\frac{\mathcal{J}_{2}(z)}{\cos \gamma}= & -\sqrt{6 \pi} \sum_{m=0}^{\infty} \frac{1}{m !(m+1) !}\left(\frac{\sqrt{R^{2}+I^{2}}}{2}\right)^{2 m+1} \\
& \times \sum_{k=0}^{m+1}\left(\begin{array}{c}
m+1 \\
k
\end{array}\right)(-1)^{k} \sum_{i=0}^{2 k}(-1)^{i}\left(\frac{1}{A}\right)^{2 k-i+1} \frac{(2 k) !}{i !} \\
& \times \begin{cases}\sinh A, & i \text { even } \\
\cosh A, & i \text { odd } .\end{cases}
\end{aligned}
$$

${ }^{1}$ B. Groh and S. Dietrich, Phys. Rev. E 50, 3814 (1994).

${ }^{2}$ H. Zhang and M. Widom, Phys. Rev. E 49, 267203 (1994).

${ }^{3}$ B. Groh and S. Dietrich, Phys. Rev. E 63, 021203 (1994).

${ }^{4}$ G. M. Range and S. H. L. Klapp, Phys. Rev. E 69, 041201 (2004).

${ }^{5}$ E. Lomba, J. J. Weis, and G. Stell, Phys Rev. E 50, 3853 (1994).

${ }^{6}$ J. M. Tavares, M. M. Telo da Gama, P. I. C. Teixeira, J. J. Weis, and M. J. P. Nijmeijer, Phys. Rev. E 52, 1915 (1995).

${ }^{7}$ A. Oukouiss and M. Baus, Phys. Rev. E 55, 7242 (1997).

${ }^{8}$ E. Lomba, J. J. Weis, and C. F. Tejero, Phys. Rev. E 58, 3426 (1998).

${ }^{9}$ F. Lado, E. Lomba, and J. J. Weis, Phys. Rev. E 58, 3478 (1998).

${ }^{10}$ M. Schoen, S. Giura, and S. H. L. Klapp, Phys. Rev. E 89, 012310 (2014).

${ }^{11}$ M. Gramzow and S. H. L. Klapp, Phys. Rev. E 75, 011605 (2007).

${ }^{12}$ I. Szalai and S. Dietrich, Eur. Phys. J. E 28, 347 (2009).

${ }^{13}$ R. Evans, Adv. Phys. 28, 143 (1979).

${ }^{14}$ S. M. Cattes, K. E. Gubbins, and M. Schoen, J. Chem. Phys. 144, 194704 (2016).

${ }^{15}$ Y. Rosenfeld, Phys. Rev. Lett. 63, 980 (1989).

${ }^{16}$ Y. Rosenfeld, D. Levesque, and J.-J. Weis, J. Chem. Phys. 92, 6818 (1990).

${ }^{17}$ P. Tarazona and R. Evans, Mol. Phys. 52, 847 (1984).

${ }^{18}$ P. Tarazona, Phys. Rev. A 31, 2672 (1985).

${ }^{19}$ A. R. Denton and N. W. Ashcroft, Phys. Rev. A 39, 4701 (1989).

${ }^{20}$ R. Leidl and H. Wagner, J. Chem. Phys. 98, 4142 (1993).

${ }^{21}$ E. Kierlik and M. L. Rosinberg, Phys. Rev. A 42, 3382 (1990).

${ }^{22}$ S. Phan, E. Kierlik, M. L. Rosinberg, B. Bildstein, and G. G. Kahl, Phys. Rev. E 48, 618 (1993).

${ }^{23}$ R. Roth, R. Evans, A. Lang, and G. Kahl, J. Phys.: Condens. Matter 14, 12063 (2002).

${ }^{24}$ R. Roth, J. Phys.: Condens. Matter 22, 063102 (2010).

${ }^{25}$ G. A. Mansoori, N. F. Carnahan, K. E. Starling, and T. W. Leland, Jr., J. Chem. Phys. 54, 1523 (1971)

${ }^{26}$ Handbook of Mathematical Functions, edited by M. Abramowitz and I. A. Stegun (Dover, New York, 1970).

${ }^{27}$ J.-P. Hansen and I. R. McDonald, Theory of Simple Liquids, 3rd ed. (Elsevier, Burlington, 2006).

${ }^{28}$ A. J. Archer and R. Evans, J. Chem. Phys. 138, 014502 (2013).

${ }^{29}$ C. G. Gray and K. E. Gubbins, Theory of Molecular Fluids (Clarendon Press, Oxford, 1984), Vol. 1.

${ }^{30}$ M. Schoen, A. J. Haslam, and G. Jackson, Langmuir 33, 11345 (2017).

${ }^{31}$ K. E. Gubbins and C. G. Gray, Mol. Phys. 23, 187 (1972).

${ }^{32}$ A. J. Archer, B. Chacko, and R. Evans, J. Chem. Phys. 147, 034501 (2017).

${ }^{33}$ R. Roth and S. Dietrich, Phys. Rev. E 62, 6926 (2000). 
${ }^{34}$ I. N. Bronštejn and K. A. Semendjaev, A Guide Book to Mathematics Fundamental Formulas-Tables-Graphs-Methods (Springer-Verlag, New York, 2012).

${ }^{35}$ S. Giura, B. G. Márkus, S. H. L. Klapp, and M. Schoen, Phys. Rev. E 87, 012313 (2013).

${ }^{36}$ S. M. Wandrei, D. G. McCarthy, and M. Schoen, Langmuir 33, 11366 (2017).

${ }^{37}$ L. Vega, E. de Miguel, L. F. Rull, G. Jackson, and I. A. McLure, J. Chem. Phys. 96, 2296 (1992).

${ }^{38}$ J. R. Henderson and F. van Swol, J. Chem. Phys. 89, 5010 (1988).
${ }^{39}$ M. Thommes and G. H. Findenegg, Langmuir 10, 4270 (1994).

${ }^{40}$ K. Morishige and M. Ito, J. Chem. Phys. 117, 8036 (2002).

${ }^{41}$ S. M. Cattes, S. H. L. Klapp, and M. Schoen, Phys. Rev. E 91, 052127 (2015).

${ }^{42}$ M. Greschek and M. Schoen, Soft Matter 6, 4931 (2010).

${ }^{43}$ J. Jordanovic and S. H. L. Klapp, Phys. Rev. Lett. 101, 038302 (2008).

${ }^{44}$ J. Jordanovic and S. H. L. Klapp, Phys. Rev. E 79, 021405 (2009).

${ }^{45}$ M. Kamalvand, T. Keshavarzi, and G. A. Mansoori, Int. J. Nanosci. 07, 245 (2008). 